Algebraic 83 Geometric $\mathcal{T}$ opology

Volume 5 (2005) 1589-1635

Published: 24 November 2005

ATG

\title{
$I$-adic towers in topology
}

\author{
SAMUEL WÜTHRICH
}

\begin{abstract}
A large variety of cohomology theories is derived from complex cobordism $M U^{*}(-)$ by localizing with respect to certain elements or by killing regular sequences in $M U_{*}$. We study the relationship between certain pairs of such theories which differ by a regular sequence, by constructing topological analogues of algebraic $I$-adic towers. These give rise to Higher Bockstein spectral sequences, which turn out to be Adams spectral sequences in an appropriate sense. Particular attention is paid to the case of completed Johnson-Wilson theory $\widehat{E(n)}$ and Morava $K$-theory $K(n)$ for a given prime $p$.
\end{abstract}

AMS Classification 55P42, 55P43, 55T15; 55U20, 55P60, 55N22

Keywords Structured ring spectra, Adams resolution, Adams spectral sequence, Bockstein operation, complex cobordism, Morava $K$-theory, Bousfield localization, stable homotopy theory.

\section{Introduction}

Complex cobordism $M U^{*}(-)$ is arguably one of the most powerful cohomology theories. It describes stable phenomena with high accuracy. A famous piece of evidence for such a vague statement are the nilpotence and periodicity theorems [29. These lead to the chromatic filtration of the stable homotopy groups of spheres. Due to its strong geometric background, the spectrum $M U$ representing complex cobordism is multiplicative in a highly structured way. Namely, $M U$ can be realized as an $E_{\infty}$ ring spectrum [25] or equivalently, in the framework of [11], as a commutative $\mathbb{S}$-algebra. As a drawback to the large amount of information that $M U^{*}(X)$ carries about a given space $X$, it is rather difficult to explicitly determine $M U^{*}(X)$ in general. The approach to confront this problem is to construct new cohomology theories by formally manipulating the theory $M U^{*}(-)$. Important possibilities are to realize regular quotients and localizations of the coefficient ring $M U_{*}$ as the coefficient rings of new theories. 
In modern geometric categories like the ones constructed in [1], it is possible to perform such constructions in a transparent way. Namely, thanks to the fact that $M U$ is a commutative $\mathbb{S}$-algebra, we may work in the monoidal and triangulated category $\mathscr{D}_{M U}$ of $M U$-modules. The monoidal structure is provided by the smash product $\wedge_{M U}$ over $M U$. Furthermore, it is possible to construct the new spectra as ring objects (monoids) in $\mathscr{D}_{M U}$, so-called $M U$-ring spectra 32 .

A natural question arises: What is the relationship among theories constructed in such a fashion? In this paper, we study the connections between two $M U-$ ring spectra $T$ and $F$, where $F$ is obtained from $T$ by killing an ideal $I$ of $T_{*}$, which is generated by a regular sequence $S$. In order to avoid technicalities, we don't make this more precise now. More generally, we consider ring spectra $T$ and $F$ over an arbitrary commutative $\mathbb{S}$-algebra $R$. An example for $R=M U$ is completed Johnson-Wilson theory $\widehat{E(n)}$ as $T$ and Morava $K$-theory $K(n)$ as $F$, for a specified prime $p$. Given a pair $T$ and $F$, we can ask whether there is a way to compute $T^{*}(X)$ from $F^{*}(X)$. If the sequence $S$ is finite there is a standard procedure to do this. Namely, we can work our way through a sequence of Bockstein spectral sequences. For the special case $\widehat{E(n)}$ and $K(n)$ for an odd prime $p$, Baker and Würgler describe in [1, 5] a different approach. They realize the $I$-adic tower under $T_{*}$

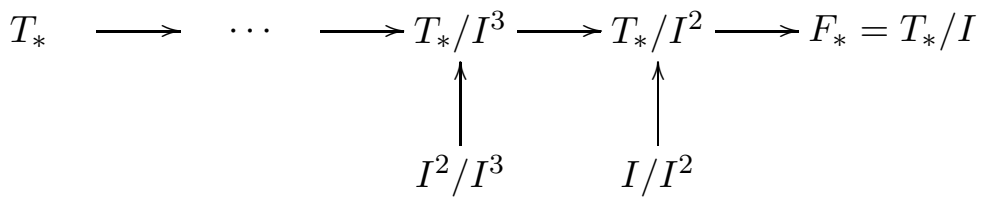

in topology and derive the so-called Higher Bockstein spectral sequence with $E_{1}$-term depending only on $F^{*}(X)$ and target $T^{*}(X)$. Based on the technology from [1], Baker and Lazarev 2] discuss a construction of $I$-adic towers for the case where the ring spectrum $T$ is a commutative $\mathbb{S}$-algebra. They note a close relationship of such towers to Adams resolutions.

The aim of this paper is to provide an alternative construction of $I$-adic towers which the author believes is more transparent and which explains their conceptual nature. This is achieved by making precise in what sense such towers are Adams resolutions. Besides the theoretical desirability of understanding the nature of $I$-adic towers, there are several practical advantages. Perhaps most importantly, the spectra $T / I^{s}$ constituting the tower become much more tractable. One concrete application the author has in mind is the study of multiplicative structures on these objects 34 . Another benefit is that it becomes possible to specify clearly what the construction depends on. It turns 
out that one can considerably weaken the assumption that the ring spectrum $T$ be strictly commutative. This will be made more explicit further below.

The strategy pursued here to develop an appropriate concept of a topological $I$-adic tower is to analyze the algebraic model in the derived setting. Namely, suppose that $T_{*}$ is commutative and consider the $I$-adic tower over $T_{*}$ in the derived category $\mathscr{D}_{T_{*}}$. It is induced by the diagram (0.1) of $T_{*}$-modules and is of the form:

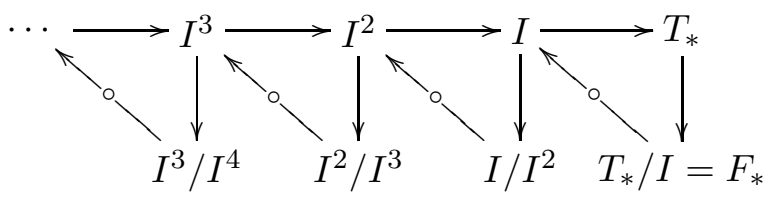

Arrows with a circle denote maps of degree -1 . The fundamental observation made in 35 , is that the sequence

$$
T_{*} \longrightarrow F_{*} \longrightarrow \mathrm{\circ} \rightarrow I / I^{2} \longrightarrow \circ I^{2} / I^{3} \longrightarrow \circ \rightarrow \cdots
$$

derived from (0.2), determines the tower up to isomorphism. The point is that (0.3) is a relative injective resolution of $T_{*}$ with respect to $F_{*}$ (in the sense of Remark [5.3) whose associated Adams resolution is given by (0.2) (Remark 6.8).

To imitate such a construction in topology, we need to have enough structure on our objects. We define the notion of a regular triple $(R, T, F)$ of ring spectra (Definition [3.6), which is meant to incorporate a rather natural and general setup in which it makes sense to consider the problem of constructing $I$-adic towers. In fact, we show that it is possible to construct such towers for any given regular triple. In the following, we motivate the ingredients which go into the definition. As mentioned before, $R$ is assumed to be a commutative $\mathbb{S}$-algebra. It plays the role of the ground ring, in the sense that we work in the category of $R$-modules $\mathscr{D}_{R}$, equipped with the smash product $\wedge_{R}$. We suppose that $T$ is a given commutative $R$-ring spectrum and that $F$ is the quotient of $T$ by a $T_{*}$-regular sequence $S=\left(x_{0}, x_{1}, \ldots\right)$ in $R_{*}$. By this, we mean that $F$ is of the form $T \wedge_{R} L$ with $L=R / x_{0} \wedge_{R} R / x_{1} \wedge_{R} \cdots$. Here $R / x_{i}$ denotes the cofibre of the multiplication map $x_{i}$, regarded as a (graded) endomorphism of $R$. Then the homotopy groups of $F$ realize the quotient $T_{*} / I$, where $I$ is the ideal generated by the image of $S$ under $\left(\eta_{T}\right)_{*}: R_{*} \rightarrow T_{*}$, induced by the unit map $\eta_{T}: R \rightarrow T$ of $T$. To obtain a manageable notion of relative injective resolutions in $\mathscr{D}_{R}$ with respect to $F$, we need $F$ to be an $R$-ring spectrum (see Remark [5.2). Rather than assuming this to be part of the data, we specify conditions which guarantee that there is an $R$-ring structure on $L$ and hence one on $F$. Namely, we assume that the coefficient $\operatorname{ring} R_{*}$ of $R$ is trivial in 
odd degrees, that the sequence $S$ is regular on $R_{*}$ and that it consists of nonzero divisors. Strickland [32] proves that these conditions are sufficient for our purpose.

In the special case $T=R$, the construction of the topological analogue of the sequence (0.3) is rather straightforward. We prove in Theorem 6.6 that it is a relative injective resolution with respect to $F$ (Definition 5.1). This allows us to define a topological $I$-adic tower as the Adams resolution associated to it. We show that its diagram of homotopy groups realizes (0.2). From there, we may construct a topological $I$-adic tower realizing (0.1):

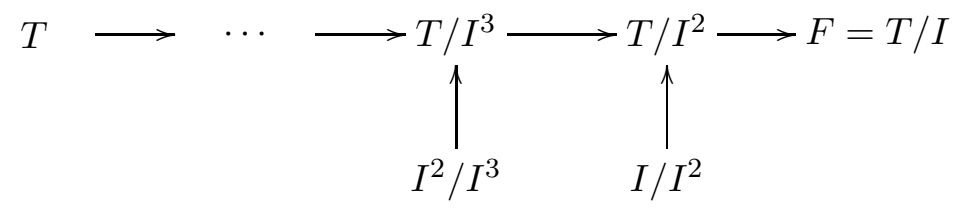

For $T \neq R$, we can do the construction above for the triple $(R, R, L)$ and apply $T \wedge_{R}-$ to it. This is an Adams resolution, and we prove that its homotopy groups realize (0.2).

If the sequence $S$ is finite, we show that the homotopy $\operatorname{limit} \widehat{T}=\operatorname{holim}_{s} T / I^{s}$ is isomorphic to the Bousfield localization $L_{F}^{R} T$ of $T$ with respect to $F$ in $\mathscr{D}_{R}$ (Proposition 6.12). If furthermore $I$ is invariant in $T_{*}(T)$, i.e. if we have $I \cdot T_{*}(T)=T_{*}(T) \cdot I$ as subgroups of the $T_{*}$-bimodule $T_{*}(T), \widehat{T}$ is isomorphic to the Bousfield localization $L_{F} T$ in the ordinary stable homotopy category. For $T=E(n)$, we identify $\widehat{E(n)}$ as the spectrum representing completed Johnson-Wilson theory from 4] (Proposition 6.18) and thus recover the result $L_{K(n)} E(n) \cong \widehat{E(n)}$ proved there.

From (0.4), we derive the Higher Bockstein spectral sequences (Theorem [7.4):

$$
\begin{aligned}
& E_{1}^{*, *}=\bigoplus_{s \geqslant 0} I^{s} / I^{s+1} \otimes_{F^{*}} F^{*}(X) \Longrightarrow \widehat{T}^{*}(X) \\
& E_{*, *}^{1}=\bigoplus_{s \geqslant 0} I^{s} / I^{s+1} \otimes_{F_{*}} F_{*}(X) \Longrightarrow\left(F_{R}^{\wedge}(T \wedge X)\right)_{*}
\end{aligned}
$$

The target of (0.6) are the homotopy groups of the so-called $F$-nilpotent completion of $T \wedge X$ in $\mathscr{D}_{R}$. We identify the first differentials as expressions in certain Bockstein operations. In case that $F$ is commutative and $F_{*}^{R}(L)$ is $F_{*}$-flat, we identify the $E_{2}$-terms as certain Coext-groups over an exterior coalgebra, which arises naturally as $F_{*}^{R}(L)$. For $T=\widehat{E(n)}$ and $F=K(n)$ for a given prime $p$, we show that the target of $[0.6)$ is $\left(L_{K(n)}(\widehat{E(n)} \wedge X)\right)_{*}$ for any $X$ (Proposition [7.6). 
The paper is organized as follows. In Section 11 we recall notation and terminology from [11]. In Section 2, we discuss duality between homology and cohomology theories represented by a not necessarily commutative $R$-ring spectrum $F$ in $\mathscr{D}_{R}$, where $R$ is a commutative $\mathbb{S}$-algebra. Furthermore, we record Künneth isomorphisms which are adapted from [6] to our situation. In Section 3. we define the notion of a regular triple, give a list of examples and do some preparatory work. In Section 4, we collect the facts that we need about algebraic $I$-adic towers from [35, 36]. In Section [5, we recall the theory of Adams resolutions and specify the injective class in $\mathscr{D}_{R}$ needed in Section [6 to construct $I$-adic towers. The construction itself is quite direct. Technically more involved is the verification that the homotopy and $F$-homology groups are as expected. Once this is done, we identify $\widehat{T}$ as a Bousfield localization in the sense and under the conditons mentioned above. We give a direct proof that for $T=E(n)$ and $I=I_{n}$, the homotopy limit $\widehat{T}$ represents completed JohnsonWilson theory, as defined in [4. In Section 17, we discuss the Higher Bockstein spectral sequences, derived from $I$-adic towers. For the identification of the $E_{2}$-terms under the conditions already mentioned, we decompose the natural $F_{*}^{R}(F)$-coaction into compatible coactions of $F_{*}^{R}(T)$ and $F_{*}^{R}(L)$. Only the coaction of the coalgebra $F_{*}^{R}(L)$ is relevant for the $E_{2}$-terms. By compar-

ing explicit descriptions of the Bousfield localization functors $\widehat{L_{K(n)}^{\widehat{E(n)}}}$ and $L_{K(n)}$ from [13] and [16] respectively, we identify the target of (0.6) for $T=\widehat{E(n)}$ and $F=K(n)$ as stated above. We end by considering some examples.

This paper forms the main part of my PhD thesis. I am deeply indebted to my supervisor Alain Jeanneret and my temporary supervisor Andrew Baker for all their encouragement and support. I would like to express my gratitude to the following persons: Urs Würgler for proposing the project to analyze Higher Bockstein spectral sequences; John Greenlees, John Rognes and Neil Strickland for helpful comments and the referee for pointing out a subtlety in the definition of regular triples. I am grateful to the Swiss National Science Foundation for financial support.

\section{Notation and terminology}

We use the framework of [1] in this paper. We start by recalling some notation and terminology from there.

The category of $\mathbb{S}$-modules $\mathscr{M}_{\mathbb{S}}$ is a symmetric monoidal model category with smash product $\wedge_{\mathbb{S}}$ and unit the sphere spectrum $\mathbb{S}$. Its homotopy category 
$\mathscr{D}_{\mathbb{S}}$, which has the same objects as $\mathscr{M}_{\mathbb{S}}$, is isomorphic to the classical stable homotopy category. When regarding an $\mathbb{S}$-module as an object of $\mathscr{D}_{\mathbb{S}}$, we call it a spectrum. The homotopy groups $\pi_{*}(E)=[\mathbb{S}, E]_{*}$ of a spectrum $E$ are written as $E_{*}$. We follow the convention $(-)^{*}=(-)_{-*}$ for graded objects. We do not distinguish a map from its suspensions in the notation.

A monoid $R$ in $\mathscr{M}_{\mathbb{S}}$ is called an $\mathbb{S}$-algebra. For any such, we have the model category of (strict) left $R$-modules $\mathscr{M}_{R}$. Its associated homotopy category $\mathscr{D}_{R}$ is a triangulated category. The graded $R^{*}$-module of morphisms between two objects $M$ and $N$ is denoted by $[M, N]_{R}^{*}$ or $N_{R}^{*}(M)$. In particular, we have $N_{*} \cong[R, N]_{R}^{-*}$. The homological degree of an element $v \in M_{*}$ is denoted by $|v|$. We also have the strict and the homotopy categories of right $R$-modules and $R$-bimodules. When speaking of an $R$-module, we mean a left $R$-module $M$, considered as an object of $\mathscr{D}_{R}$. When we view $M$ as an object in $\mathscr{M}_{R}$, we refer to it as a strict $R$-module.

The smash product $\wedge_{R}$ over $R$ is a bifunctor, which assigns to a strict right and a strict left $R$-module $M$ and $N$ respectively an $\mathbb{S}$-module $M \wedge_{R} N$. The function $R$-module functor sends a pair $N$ and $L$ of strict left $R$-modules to an $\mathbb{S}$-module $F_{R}(N, L)$. There is an obvious adjunction formula between $\wedge_{R}$ and $F_{R}$. Both functors descend to homotopy categories. We have $\pi_{-*}\left(F_{R}(N, L)\right) \cong$ $[N, L]_{R}^{*}$ and write $M_{*}^{R}(N)$ for $\pi_{*}\left(M \wedge_{R} N\right)$. The functors $L_{R}^{*}(-)$ and $M_{*}^{R}(-)$ define cohomology and homology functors on $\mathscr{D}_{R}$ respectively. If $R$ is clear from the context, we refer to them as $L$-cohomology and $M$-homology.

The category of strict $R$-bimodules is a monoidal model category; hence its homotopy category is monoidal. If $R$ is a commutative $\mathbb{S}$-algebra, the strict categories of left and right $R$-modules are isomorphic and every strict one-sided module is a strict bimodule. Hence $\wedge_{R}$ gives rise to a monoidal model category structure on $\mathscr{M}_{R}$ and therefore to a monoidal structure on $\mathscr{D}_{R}$. Monoids in $\mathscr{M}_{R}$ and $\mathscr{D}_{R}$ are called $R$-algebras and $R$-ring spectra respectively.

If $T$ is an $R$-ring spectrum, we may consider algebras over the monad [23, VI] $T \wedge_{R}-$ in $\mathscr{D}_{R}$. These are objects $M$ of $\mathscr{D}_{R}$ with an action map $T \wedge_{R} M \rightarrow M$ in $\mathscr{D}_{R}$, satisfying the associativity and unit axioms. We refer to such $M$ as $T$-module spectra.

For a commutative $\mathbb{S}$-algebra $R$ and an $R$-module $M$, multiplication by an element $x \in R_{*}$ defines a map $\Sigma^{|x|} M \stackrel{x}{\rightarrow} M$ in $\mathscr{D}_{R}$. We use the notation

$$
\Sigma^{|x|} M \stackrel{v}{\longrightarrow} M \stackrel{\rho_{x}}{\longrightarrow} M / x \stackrel{\beta_{x}}{\longrightarrow} \Sigma^{|x|+1} M
$$

from [32 for its cofibre and the maps to and from it. Note that $M / x \cong$ 
$M \wedge_{R} R / x$. Furthermore, we define $\bar{\beta}_{x}$ to be the composition

$$
\bar{\beta}_{x}=\rho_{x} \circ \beta_{x}: M / x \longrightarrow \Sigma^{|x|+1} M / x .
$$

For a sequence $S=\left(x_{1}, x_{2}, \ldots\right)$ of elements of $R_{*}$, we write

$$
M / S=M \wedge \bigwedge_{j} R / x_{j}
$$

and call $M / S$ a quotient of $M$. Here, as well as throughout the paper, $\wedge$ denotes $\wedge_{R}$, where $R$ is specified in the context. The symbol $*$ denotes a point. A blank $\otimes$ means $\otimes_{F_{*}}$, where $F_{*}$ is the coefficient ring of some ring spectrum $F$ under consideration. The category of $F_{*}$-modules is denoted by $\operatorname{Mod}_{F *}$.

\section{Künneth isomorphisms for $R$-ring spectra}

Let $R$ be a commutative $\mathbb{S}$-algebra and let $F$ be an $R$-ring spectrum. We can define an exterior product

$$
\times: F_{*}^{R}(M) \otimes F_{*}^{R}(N) \longrightarrow F_{*}^{R}(M \wedge N)
$$

for given $R$-modules $M$ and $N$ in the usual way. However, as $F$ is not assumed to be commutative, we need to take care with the right action of $F_{*}$ on $F_{*}^{R}(M)$.

For $x \in F_{*}^{R}(M)$ and $\gamma \in F_{*}, x \cdot \gamma$ is defined as the composition:

$$
R \stackrel{x}{\longrightarrow} F \wedge M \cong F \wedge R \wedge M \stackrel{F \wedge \gamma \wedge M}{\longrightarrow} F \wedge F \wedge M \stackrel{\mu \wedge M}{\longrightarrow} F \wedge M
$$

The following fact is well-known for commutative $F$, see [6. Thm. 4.2] for instance. The argument generalizes to our situation. The point is that the smallest localizing subcategory of $\mathscr{D}_{R}$ [15, Def. 1.1.1] generated by the suspensions of $R$ is $\mathscr{D}_{R}$.

Proposition 2.1 Assume that $F_{*}^{R}(M)$ or $F_{*}^{R}(N)$ is $F_{*}$-flat. Then the pairing (2.1) induces the Künneth isomorphism of $F_{*}$-modules

$$
F_{*}^{R}(M) \otimes F_{*}^{R}(N) \cong F_{*}^{R}(M \wedge N) .
$$

In the usual way, we construct a Kronecker pairing

$$
F_{*}^{R}(M) \otimes F_{R}^{*}(M) \longrightarrow F_{*}
$$

of left $F_{*}$-modules. We use the same right $F_{*}$-action on $F_{*}^{R}(M)$ as in (2.1) to form the tensor product. Adjoint to the Kronecker pairing is a duality morphism

$$
F_{R}^{*}(M) \longrightarrow \operatorname{Hom}_{F_{*}}\left(F_{*}^{R}(M), F_{*}\right)
$$


of left $F_{*}$-modules. We abbreviate $\operatorname{Hom}_{F_{*}}\left(-, F_{*}\right)$ by $D(-)$ in the following.

In order to obtain Künneth isomorphisms in cohomology, we need to consider a completed version of cohomology, analogous to the one considered in [6]. First, we need an analogue of the profinite filtration of cohomology groups as used in 6. Def. 4.9]. Our objects, the $R$-modules, are not naturally built from cells, but we can approximate them by such objects. Recall from [11, III.2] that a cell $R$-module is a strict $R$-module $M$ which comes with a certain filtration, called the sequential filtration. It consists of sub $R$-modules $M_{n}$ with $M_{0}=*$ and with the property that $M_{n+1}$ is obtained from $M_{n}$ by attaching a wedge of $R$-spheres. Together with the $R$-linear maps which respect this filtration, the cell $R$-modules form a subcategory of $\mathscr{M}_{R}$. The approximation theorem [11, Thm. III.2.10] states that for each strict $R$-module $M$ there exists a cell $R$-module $\Gamma M$ and a weak equivalence $\gamma: \Gamma M \rightarrow M$. The pair $(\Gamma M, \gamma)$ is called a cell approximation of $M$. With this background, we are in a position where we can mimick the definitions from [6].

Definition 2.2 Let $M$ be a cell $R$-module. The profinite filtration of $F_{R}^{*}(M)$ consists of all the ideals

$$
F^{a} F_{R}^{*}(M)=\operatorname{ker}\left(F_{R}^{*}(M) \longrightarrow F_{R}^{*}\left(M_{a}\right)\right)
$$

where $M_{a}$ runs through all finite cell submodules of $M$. These ideals form a directed system. For an arbitrary $R$-module $M$, choose a cell approximation $(\Gamma M, \gamma)$. The profinite filtration on $F_{R}^{*}(\Gamma M)$ induces a filtration on $F_{R}^{*}(M)$ via the isomorphism

$$
F_{R}^{*}(\gamma): F_{R}^{*}(M) \cong F_{R}^{*}(\Gamma M) .
$$

Define the profinite topology on $F_{R}^{*}(M)$ to be the induced $F^{*}$-linear topology.

As a consequence of [11, Lemma III.2.2], the profinite topology is independent of the choice of a cell approximation.

Definition 2.3 For an $R$-module $M$, completed $F$-cohomology $F_{R}^{*}(M)^{\wedge}$ is defined as the completion of $F_{R}^{*}(M)$ with respect to the profinite topology.

Definition 2.4 Let $M_{*}$ be an $F_{*}-$ module. The dual-finite filtration on $D M_{*}$ consists of the submodules

$$
F^{L}\left(D M_{*}\right)=\operatorname{ker}\left(D M_{*} \longrightarrow D L_{*}\right),
$$

where $L_{*}$ runs through all finitely generated submodules of $M_{*}$. It gives rise to the dual-finite topology on $D M_{*}$. 
The duality morphism (2.2) is continuous with respect to the profinite and the dual-finite topologies respectively. We can prove the following statement in the same way as [6, Thm. 4.14].

Proposition 2.5 Let $M$ be an $R$-module for which $F_{*}^{R}(M)$ is $F_{*}$-free. Then the duality morphism induces a homeomorphism

$$
F_{R}^{*}(M) \cong D\left(F_{*}^{R}(M)\right) .
$$

In cohomology, we have an exterior product

$$
F_{R}^{*}(M) \otimes F_{R}^{*}(N) \longrightarrow F_{R}^{*}(M \wedge N)
$$

defined as usual. Again, we have to be careful with the right action of $F_{*}$. For $x \in F_{R}^{*}(M)$ and $\gamma \in F_{*}, x \cdot \gamma$ is defined as

$$
M \stackrel{x}{\longrightarrow} F \cong F \wedge R \stackrel{F \wedge \gamma}{\longrightarrow} F \wedge F \stackrel{\mu}{\longrightarrow} F .
$$

As cell structures on $M$ and on $N$ induce one on $M \wedge N$ [11, Prop. III.7.3], the product (2.3) lifts to a pairing

$$
F_{R}^{*}(M) \widehat{\otimes} F_{R}^{*}(N) \longrightarrow F_{R}^{*}(M \wedge N)^{\wedge} .
$$

Here, the left hand side is the completion with respect to the canonical topology on the tensor product. The following Künneth-type theorem can be proved in the same way as [6, Thm. 4.19].

Proposition 2.6 Assume that $F_{*}^{R}(M)$ is $F_{*}$-free. If $F_{*}^{R}(M)$ is finitely generated or if $F_{*}^{R}(N)$ is $F_{*}$-free, there is an isomorphism of left $F^{*}$-modules

$$
F_{R}^{*}(M \wedge N) \cong F_{R}^{*}(M) \widehat{\otimes} F_{R}^{*}(N) .
$$

\section{$3 \quad$ Regular triples of ring spectra}

Let $M$ be an $R$-module. Recall the quotient constructions $M / x$ and $M / S$ from Section 1 defined for an element $x$ and a sequence of elements $S$ in $R_{*}$ respectively.

Definition 3.1 If $S=\left(x_{1}, x_{2}, \ldots\right)$ is regular on $M_{*}$, we call $M / S$ a regular quotient of $M$. Explicitly, this means that multiplication by $x_{i}$ is injective on $M_{*} /\left(x_{1}, \ldots, x_{i-1}\right) M_{*}$ and that $M_{*} / S M_{*} \neq 0$. 
Remark 3.2 If $S$ and $S^{\prime}$ are regular sequences generating the same ideal $I$, the regular quotients $M / S$ and $M / S^{\prime}$ are isomorphic [11, Cor. V.2.10]. So it suffices to specify an ideal $I$ and set $M / I=M / S$ for some regular sequence $S$ generating $I$.

Let $F$ be some $R$-ring spectrum and let $N=M / S$ be a quotient of $M$ with respect to a sequence $S=\left(x_{0}, x_{1}, \ldots\right)$ in $R_{*}$. Let $\Lambda_{F_{*}}\left(\alpha_{0}, \alpha_{1}, \ldots\right)$ denote the exterior algebra on a set of generators $\alpha_{j}$ in bijective correspondence with the $x_{j}$, with degrees $\left|\alpha_{j}\right|=\left|x_{j}\right|+1$. Its $F_{*}$-dual is the completed exterior algebra $\widehat{\Lambda}_{F_{*}}\left(\alpha_{0}^{\vee}, \alpha_{1}^{\vee}, \ldots\right)$ on the dual basis $\left\{\alpha_{j}^{\vee}\right\}$ of $\left\{\alpha_{j}\right\}$. The statement below follows from Propositions 2.1] and 2.6.

Lemma 3.3 Assume that $F_{*}^{R}(M)$ is $F_{*}$-free and that the elements $x_{j}$ are contained in the annihilator of $F_{*}$. Then there are isomorphisms of $F_{*}$-modules:

$$
\begin{aligned}
& F_{*}^{R}(N) \cong F_{*}^{R}(M) \otimes F_{*}^{R}\left(\bigwedge_{j} R / x_{j}\right) \cong F_{*}^{R}(M) \otimes \Lambda_{F_{*}}\left(\alpha_{0}, \alpha_{1}, \ldots\right) \\
& F_{R}^{*}(N) \cong F_{R}^{*}(M) \widehat{\otimes} F_{R}^{*}\left(\bigwedge_{j} R / x_{j}\right) \cong F_{R}^{*}(M) \widehat{\otimes} \widehat{\Lambda}_{F^{*}}\left(\alpha_{0}^{\vee}, \alpha_{1}^{\vee}, \ldots\right)
\end{aligned}
$$

Let now $\left(x_{0}, x_{1}, \ldots\right)$ be a regular sequence on $R_{*}$ consisting of non-zero divisors. Assume that $R$ is even, by which we mean that the homotopy $R_{*}$ is concentrated in even degrees. Then Strickland proves in [32, Prop. 3.1] that the $R$-modules $R / x_{j}$ can be realized as $R$-ring spectra. Fix such products. Then there is a unique $R$-ring structure on $L=\bigwedge_{j} R / x_{j}$ such that the maps $R / x_{j} \rightarrow L$ are commuting ring maps, in the sense of [32, Def. 4.1].

Definition 3.4 Let $R$ be even. A regular $R$-ring is a regular quotient of $R$ with respect to some regular sequence on $R_{*}$ consisting of non-zero divisors, together with some chosen $R$-ring structure arising as above.

Let $L$ be a regular $R$-ring. Smashing a map from $\left(R / x_{j}\right)_{R}^{*}\left(R / x_{j}\right)$ with the identities on the other smash factors yields a homomorphism of $R_{*}$-algebras

$$
q_{j}:\left(R / x_{j}\right)_{R}^{*}\left(R / x_{j}\right) \longrightarrow L_{R}^{*}(L) .
$$

Lemma 3.3 implies that $\left(R / x_{j}\right)_{R}^{*}\left(R / x_{j}\right)$ and $L_{R}^{*}(L)$ are free over $R^{*} /\left(x_{j}\right)$ and $L^{*}$ respectively. Hence they admit coalgebra structures. Clearly, the $q_{j}$ are coalgebra maps. Let $\bar{\beta}_{j}$ be the map $\bar{\beta}_{x_{j}}$ as defined in (1.2). Denote the image $q_{j}\left(\bar{\beta}_{j}\right) \in L_{R}^{*}(L)$ by $Q_{j}$. 
Proposition 3.5 Let $R$ be even. If $L$ is a regular $R$-ring, there is an isomorphism of $L_{*}-$ modules

$$
L_{*}^{R}(L) \cong \Lambda_{L_{*}}\left(a_{0}, a_{1}, \ldots\right)
$$

and an isomorphism of $L_{*}$-bialgebras

$$
L_{R}^{*}(L) \cong \widehat{\Lambda}_{L^{*}}\left(Q_{0}, Q_{1}, \ldots\right)
$$

where $\left|a_{j}\right|=\left|Q_{j}\right|=\left|x_{j}\right|+1$.

Proof It follows from Lemma 3.3 that the module structures are as asserted. For the identification of $L_{R}^{*}(L)$ as an algebra see [32, Prop. 4.15]. The coalgebra structure is determined by the fact that the $Q_{j}$ are derivations [32, Cor. 4.19] and hence are primitive with respect to the coproduct.

Definition 3.6 Suppose that $R$ is an even commutative $\mathbb{S}$-algebra and that $T$ is a commutative $R$-ring spectrum. Assume that $F$ is an $R$-ring spectrum of the form $F=T \wedge L$, where $L$ is a regular $R$-ring with respect to a sequence $S=\left(x_{0}, x_{1}, \ldots\right)$ in $R_{*}$ which is regular both on $R_{*}$ and on $T_{*}$. Then we call $(R, T, F)$ a regular triple.

We write $J=\left(x_{0}, x_{1}, \ldots\right) \triangleleft R_{*}$ and $I=J \cdot T_{*} \triangleleft T_{*}$ for the ideals generated by $S$ in the rings $R_{*}$ and $T_{*}$ respectively. Thus we have $L_{*} \cong R_{*} / J$ and $F_{*} \cong T_{*} / I$. We abuse notation and write $x_{j}$ for the image in $T_{*}$ of the elements $x_{j} \in R_{*}$ under $\left(\eta_{T}\right)_{*}: R_{*} \rightarrow T_{*}$, induced by the unit $\eta_{T}: R \rightarrow T$ of $T$.

Remark 3.7 If 2 is a unit in $L_{*}, L$ may be realized as a commutative $R$-ring spectrum [32, Thm. 4.11]. So $F$ is then commutative as an $R$-ring spectrum.

The following is clear from the statements in Section 2 and Proposition 3.5 . Because the maps $L_{*}^{R}(L) \rightarrow F_{*}^{R}(L)$ and $L_{R}^{*}(L) \rightarrow F_{R}^{*}(L)$ induce isomorphisms on tensoring the sources with $F_{*}$, we don't introduce new labels for the generators of $F_{*}^{R}(L)$ and $F_{R}^{*}(L)$. The condition that $F_{*}^{R}(T)$ is $F_{*}$-free is always satisfied if $T$ is a regular quotient of $R$, as a consequence of Lemma 3.3.

Lemma 3.8 Let $(R, T, F)$ be a regular triple. Then there is an isomorphism of $F_{*}$-modules

$$
F_{*}^{R}(F) \cong F_{*}^{R}(T) \otimes \Lambda_{F_{*}}\left(a_{0}, a_{1}, \ldots\right) .
$$

If the sequence $S$ generating $I$ is finite or if $F_{*}^{R}(T)$ is $F_{*}$-free, we have

$$
F_{R}^{*}(F) \cong F_{R}^{*}(T) \widehat{\otimes} \Lambda_{F^{*}}\left(Q_{0}, Q_{1}, \ldots\right)
$$


as $F^{*}$-modules. In this case, the exterior algebra $F^{*} \otimes L_{R}^{*}(L)$ maps isomorphically onto the subalgebra $\Lambda_{F^{*}}\left(Q_{0}, Q_{1}, \ldots\right)$ under the natural map of $F^{*}-$ algebras

$$
F^{*} \otimes L_{R}^{*}(L) \longrightarrow F_{R}^{*}(F)
$$

Recall the various $M U$-modules $v_{n}^{-1} B P, P(n), B(n), B P\langle n\rangle, E(n), \widehat{E(n)}$, $k(n), K(n)$ derived from the Brown-Peterson spectrum $B P$ for some prime $p$, with

$$
B P_{*} \cong \mathbb{Z}_{(p)}\left[v_{1}, v_{2}, \ldots\right]
$$

where $\left|v_{n}\right|=2\left(p^{n}-1\right)$ (see [28] for instance). Let $I_{n}=\left(v_{0}, \ldots, v_{n-1}\right)$, where as usual $v_{0}=p$. We have isomorphisms of $B P_{*}-$ modules

$$
\begin{aligned}
P(n)_{*} & \cong \mathbb{F}_{p}\left[v_{n}, v_{n+1}, \ldots\right] & & B(n)_{*} \cong v_{n}^{-1} P(n)_{*} \\
B P\langle n\rangle_{*} & \cong \mathbb{Z}_{(p)}\left[v_{1}, \ldots, v_{n}\right] & & E(n)_{*} \cong v_{n}^{-1} B P\langle n\rangle_{*} \\
\left(v_{n}^{-1} B P\right)_{*} & \cong v_{n}^{-1} B P_{*} & & \widehat{E(n)_{*}} \cong\left(E(n)_{*}\right)_{I_{n}} \\
k(n)_{*} & \cong \mathbb{F}_{p}\left[v_{n}\right] & & K(n)_{*} \cong v_{n}^{-1} k(n)_{*}
\end{aligned}
$$

where $(-)_{I_{n}}^{\wedge}$ is completion at $I_{n}$. Using results from [32, we find the following examples of regular triples:

Example 3.9 $R=M U, T=E(n)$ and $F=K(n)$ for an odd prime $p$. For $p=2$, the substitute $E(n)^{\prime}$ is commutative, see 32 . Hence $\left(M U, E(n)^{\prime}, K(n)\right)$ is regular.

Example 3.10 From [3] or [12] we know that $\widehat{E(n)}$ is a commutative $\mathbb{S}$ algebra. So $(\widehat{E(n)}, \widehat{E(n)}, K(n))$ is a regular triple. Of course, $(M U, \widehat{E(n)}, K(n))$ is regular as well.

Example 3.11 $R=M U, T=B P, F=P(n)$.

Example 3.12 $R=M U, T=P(n), F=k(n)$ for odd $p$.

Example 3.13 $R=M U, T=B P\langle n\rangle, F=k(n)$ for odd $p$. For $p=2$, we may take $B P\langle n\rangle^{\prime}$ instead, see [32].

Example 3.14 $R=v_{n}^{-1} M U, T=v_{n}^{-1} B P, F=K(n)$.

Example 3.15 $R=v_{n}^{-1} M U, T=B(n), F=K(n)$ for odd $p$. 
We end the section by deriving a characterization of the morphisms $L_{*}^{R}\left(Q_{j}\right)$ induced by the Bockstein operations on $L$-homology groups. By definition, the morphism induced by $Q_{j}$ on $L$-cohomology

$$
L_{R}^{*}\left(Q_{j}\right): L_{R}^{*}(L) \longrightarrow L_{R}^{*+\left|x_{j}\right|+1}(L)
$$

is multiplication by $Q_{j}$ on the right. In the following $D(-)$ denotes the continuous $L_{*}$-duality operator. Consider

$$
D\left(L_{R}^{*}\left(Q_{j}\right)\right): D\left(L_{R}^{*+\left|x_{j}\right|+1}(L)\right) \longrightarrow D\left(L_{R}^{*}(L)\right) .
$$

The coalgebra structure on $L_{R}^{*}(L)$ induces an algebra structure on $D\left(L_{R}^{*}(L)\right)$, which is just the completed exterior algebra generated by the dual basis $\left\{Q_{j}^{\vee}\right\}$ of $\left\{Q_{j}\right\}$. Because $L_{R}^{*}\left(Q_{j}\right)$ is multiplication by a primitive element, the dual map $D\left(L_{R}^{*}\left(Q_{j}\right)\right)$ is a derivation with respect to this algebra structure. As such, it is determined by

$$
D\left(L_{R}^{*}\left(Q_{j}\right)\right)\left(Q_{i}^{\vee}\right)=\delta_{i j},
$$

where $\delta_{i j}$ is the Kronecker delta. Now as $L_{*}^{R}(L)$ is a free $L_{*}-\operatorname{module}, L_{*}^{R}(L)$ injects into its bidual $D D\left(L_{*}^{R}(L)\right)$ and $D D\left(L_{*}^{R}\left(Q_{j}\right)\right)$ restricts to $L_{*}^{R}\left(Q_{j}\right)$. Via the duality isomorphism

$$
L_{R}^{*}(L) \cong D\left(L_{*}^{R}(L)\right)
$$

the morphism $D\left(L_{*}^{R}\left(Q_{j}\right)\right)$ corresponds to $L_{R}^{*}\left(Q_{j}\right)$, and therefore (3.2) describes $L_{*}^{R}\left(Q_{j}\right)$. Hence, choosing the $a_{j} \in L_{*}^{R}(L)$ and their formal exterior products so that they correspond to the $Q_{j}^{\vee}$ and their products, we have shown the following.

Lemma 3.16 Endow $L_{*}^{R}(L) \cong \Lambda_{L_{*}}\left(a_{0}, a_{1}, \ldots\right)$ with the exterior algebra structure. Then the homomorphism

$$
L_{*}^{R}\left(Q_{j}\right): L_{*}^{R}(L) \longrightarrow L_{*-\left|x_{j}\right|-1}^{R}(L)
$$

is the derivation $\frac{\partial}{\partial a_{j}}$ characterized by $\frac{\partial}{\partial a_{j}}\left(a_{i}\right)=\delta_{i j}$.

Remark 3.17 If $L$ is commutative, the algebra structure of $L_{*}^{R}(L)$ induced by the multiplication on $L$ is the one considered in the lemma. This follows from the fact that the duality map $d: L_{R}^{*}(L) \cong D\left(L_{*}^{R}(L)\right)$ is an algebra map under this assumption. For non-commutative $L$, the two algebra structures will not agree in general. 


\section{The algebra of $I$-adic towers}

Let $(R, T, F)$ be a regular triple with $F \cong T \wedge L$. Recall the isomorphisms $L_{*} \cong$ $R_{*} / J$ and $F_{*} \cong T_{*} / I$, where $J$ is generated by a regular sequence $\left(x_{0}, x_{1}, \ldots\right)$ and $I=J \cdot T_{*}$. The contents of this section apply to both $\left(R_{*}, J_{*}\right)$ and $\left(T_{*}, I_{*}\right)$. We formulate everything for the first case and write $\otimes$ for $\otimes_{L_{*}}$ in this section.

Define two families $\left(\mathcal{E}^{s}\right)_{s \geqslant 0}$ and $\left(\mathcal{F}^{s}\right)_{s \geqslant 0}$ of short exact sequences of $R_{*}$-modules

$$
\begin{array}{ll}
\mathcal{E}^{s}: & 0 \longrightarrow J^{s+1} \stackrel{i_{s+1}}{\longrightarrow} J^{s} \stackrel{p_{s}}{\longrightarrow} J^{s} / J^{s+1} \longrightarrow 0 \\
\mathcal{F}^{s}: & 0 \longrightarrow J^{s} / J^{s+1} \stackrel{j_{s}}{\longrightarrow} R_{*} / J^{s+1} \stackrel{q_{s+1}}{\longrightarrow} R_{*} / J^{s} \longrightarrow 0
\end{array}
$$

where by convention $J^{0}=R_{*}$. All the maps are the canonical ones. Whenever it is clear from the context, we will omit the index $s$. We will also write $i$ and $q$ for a composition of maps $i$ and $q$ as defined above.

Let $\mathscr{D}_{R_{*}}$ be the derived category of the ring $R_{*}$. Its objects are the chain complexes of graded $R_{*}$-modules. The sequences $\mathcal{E}^{s}$ and $\mathcal{F}^{s}$ induce towers in $\mathscr{D}_{R_{*}}$ consisting of triangles, the $J$-adic tower over $R_{*}$

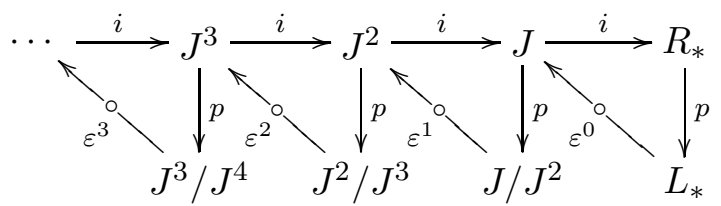

and the $J$-adic tower under $R_{*}$ :

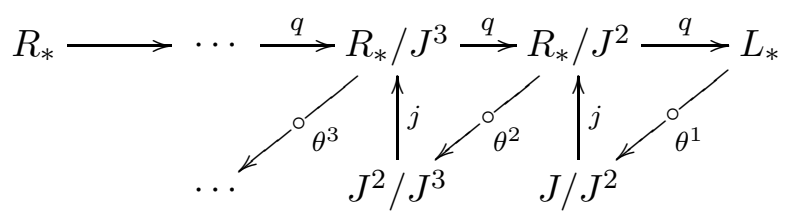

Arrows with a circle denote morphisms of degree -1 .

Let $M_{*}$ be a graded $R_{*}$-module. We write $\operatorname{gr}_{J}^{*}\left(M_{*}\right)$ for the (bi)graded $L_{*}-$ module associated to the $J$-adic filtration on $M_{*}$. For $M_{*}=R_{*}$, we have

$$
\operatorname{gr}_{J}^{*}\left(R_{*}\right)=\bigoplus_{s \geqslant 0} J^{s} / J^{s+1} .
$$

For a graded $L_{*}$-module $Y_{*}, \operatorname{Sym}_{L_{*}}^{*}\left(Y_{*}\right)$ denotes the symmetric algebra over $L_{*}$ on $Y_{*}$. It is naturally bigraded. 
Proposition 4.1 (24, Thm. 16.2]) The $L_{*}-$ module $J / J^{2}$ is freely generated by the residue classes $\left\{x_{j}\right\}$ of the generators $x_{j} \in J$. Furthermore, we have an isomorphism of graded $L_{*}$-algebras

$$
\operatorname{gr}_{J}^{*}\left(R_{*}\right) \cong \operatorname{Sym}_{L_{*}}^{*}\left(J / J^{2}\right) .
$$

It follows that we have isomorphisms of $L^{*}$-modules

$$
\operatorname{Ext}_{R^{*}}^{*, *}\left(L^{*}, J^{s} / J^{s+1}\right) \cong \operatorname{Ext}_{R^{*}}^{*, *}\left(L^{*}, L^{*}\right) \otimes J^{s} / J^{s+1} .
$$

The element of $\operatorname{Ext}_{R^{*}}^{1,0}\left(L^{*}, J / J^{2}\right)$ associated to the short exact sequence

$$
\mathcal{F}^{1}: \quad 0 \longrightarrow J / J^{2} \longrightarrow R_{*} / J^{2} \longrightarrow L_{*} \longrightarrow 0
$$

corresponds via (4.3) to a sum of the form $\sum f_{j} \otimes\left\{x_{j}\right\}$ with

$$
f_{j} \in \operatorname{Ext}_{R^{*}}^{1,-\left|x_{j}\right|}\left(L^{*}, L^{*}\right) .
$$

The following fact is as well-known as fundamental, see [24, Thm. 16.5] and for the multiplicative structure [33, §4.5]. The identification of the elements $f_{j}$ as generators is straight-forward.

Proposition 4.2 There are isomorphisms of $L_{*}$-algebras

$$
\begin{aligned}
& \operatorname{Ext}_{R^{*}}^{*, *}\left(L^{*}, L^{*}\right) \cong \widehat{\Lambda}_{L^{*}}\left(f_{0}, f_{1}, \ldots\right) \\
& \operatorname{Tor}_{*, *}^{R_{*}}\left(L_{*}, L_{*}\right) \cong \Lambda_{L_{*}}\left(e_{0}, e_{1}, \ldots\right)
\end{aligned}
$$

where the $f_{j}$ and the $e_{j}$ have bidegree $\left(1,-\left|x_{j}\right|\right)$ and $\left(1,\left|x_{j}\right|\right)$ respectively.

Let $\otimes^{\mathbf{L}}$ and $\mathbf{R H o m}$ be the left and right derived functors of $\otimes_{R_{*}}$ and $\operatorname{Hom}_{R_{*}}$ respectively. The functors

$$
H L_{p, *}(-)=H_{p}\left(L_{*} \otimes^{\mathbf{L}}-\right), \quad H L^{p, *}(-)=H^{p}\left(\mathbf{R H o m}\left(-, L^{*}\right)\right)
$$

define a homology and a cohomology theory on $\mathscr{D}_{R_{*}}$ respectively. We call them $L_{*}$-homology and $L_{*}$-cohomology. Both $H L_{*, *}(-)$ and $H L^{*, *}(-)$ take values in the category of $L_{*}$-modules. Their restriction to $R_{*}$-modules are the functors $\operatorname{Tor}_{*, *}^{R_{*}}\left(L_{*},-\right)$ and $\operatorname{Ext}_{R^{*}}^{*, *}\left(-, L^{*}\right)$ respectively.

It is well-known that the cohomology theory $E^{*}(-)$ takes values in $E^{*}(E)-$ modules if $E$ is a ring spectrum. Also, if $E_{*}(E)$ is $E_{*}$-flat, $E_{*}(E)$ has a natural coalgebra structure and $E_{*}(-)$ takes values in $E_{*}(E)$-comodules. Mimicking the definition of these structures, we can define a natural coproduct on $H L_{*, *}\left(L_{*}, L_{*}\right)$ and show that the functors $H L_{*, *}(-)$ and $H L^{*, *}(-)$ take values in the categories of $H L_{*, *}\left(L_{*}\right)$-comodules and $H L^{*, *}\left(L^{*}\right)$-modules respectively. Arguing as in Remark 3.17 we find that the product on $H L^{*, *}\left(L^{*}\right)$ is dual to the 
coproduct on $H L_{*, *}\left(L_{*}\right)$. Hence the latter agrees with the natural coproduct on $\Lambda_{L_{*}}\left(e_{0}, e_{1}, \ldots\right)$.

The following result, proved as [36, Thm. 3.8] or [35. Thm. 1], describes how surprisingly simple the $L_{*}$-homology of the $J$-adic tower over $R_{*}$ is. In 36 and 35, we only considered finite regular quotients. The general case, however, follows easily by passing to limits.

Theorem 4.3 The functor $H L_{*, *}(-)$ maps the algebraic $J$-adic tower over $R_{*}$ to a diagram of $H L_{*, *}\left(L_{*}\right)$-comodules composed of short exact sequences of the form

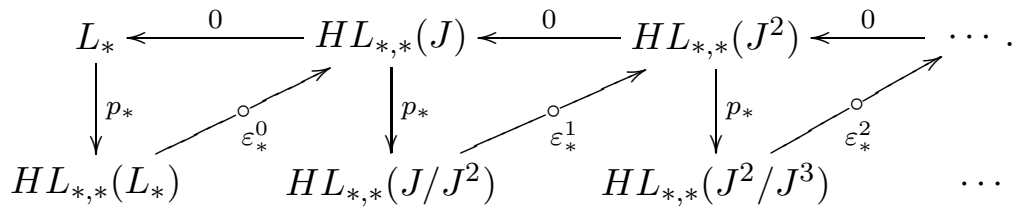

Furthermore, all modules are free over $L_{*}$.

From this, we easily derive the $L_{*}$-homology of the $J$-adic tower under $R_{*}$. Namely, we have an isomorphism $H L_{*, *}\left(R_{*} / J^{s}\right) \cong L_{*} \oplus H L_{*-1, *}\left(J^{s}\right)$, induced by the long exact sequence of homology groups associated to

$$
0 \longrightarrow J^{s} \stackrel{i}{\longrightarrow} R_{*} \stackrel{q}{\longrightarrow} R_{*} / J^{s} \longrightarrow 0 .
$$

We set

$$
\widetilde{H L}_{*, *}\left(R_{*} / J^{s}\right)=\operatorname{ker}\left(H L_{*, *}\left(R_{*} / J^{s}\right) \rightarrow L_{*}\right) \cong H L_{*-1, *}\left(J^{s}\right) .
$$

Corollary 4.4 Applying $H L_{*, *}(-)$ to the algebraic $J$-adic tower under $R_{*}$ yields an unrolled exact couple of the form:

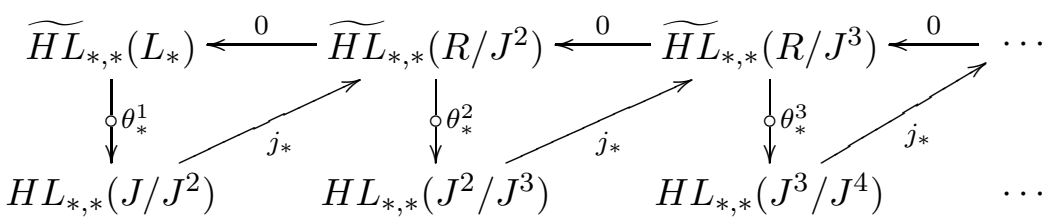

Furthermore, all modules are free over $L_{*}$.

Proof Let $\varphi^{s}$ be the connecting homomorphism associated to (4.5). By naturality of connecting homomorphisms, we have a commutative diagram:

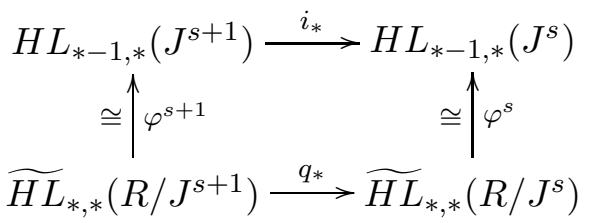


Hence the theorem implies that $\widetilde{H L}_{*, *}\left(R / J^{s+1}\right) \stackrel{q_{*}}{\longrightarrow} \widetilde{H L}_{*, *}\left(R / J^{s}\right)$ is trivial.

Remark 4.5 As all homology groups appearing in Theorem 4.3 and Corollary 4.4 are $L_{*}$-free, the $L_{*}$-cohomology of the algebraic $J$-adic towers is isomorphic to the $L_{*}$-dual of their respective $L_{*}$-homology. This follows from a statement analogous to Proposition 2.5. but for the algebraic derived category $\mathscr{D}_{R_{*}}$.

By Proposition 4.1, there are isomorphisms of $L_{*}-$ modules

$$
H L_{*, *}\left(J^{s} / J^{s+1}\right) \cong H L_{*, *}\left(L_{*}\right) \otimes J^{s} / J^{s+1} .
$$

In fact, these are isomorphisms of $H L_{*, *}\left(L_{*}\right)$-comodules, if we regard the right hand side as the cofree $H L_{*, *}\left(L_{*}\right)$-comodule generated by $J^{s} / J^{s+1}$. As all modules in (4.4) are $L_{*}$-free, we obtain a long exact sequence with the following property (see [22, Ch. IX] for a discussion of relative homological algebra):

Corollary 4.6 The sequence of $H L_{*, *}\left(L_{*}\right)$-comodules

$0 \rightarrow L_{*} \stackrel{p_{*}}{\longrightarrow} H L_{*, *}\left(L_{*}\right) \stackrel{\delta^{0}}{\longrightarrow} H L_{*-1, *}\left(L_{*}\right) \otimes J / J^{2} \stackrel{\delta^{1}}{\longrightarrow} H L_{*-2, *}\left(L_{*}\right) \otimes J^{2} / J^{3} \rightarrow \cdots$, where $\delta^{s}=p_{*} \varepsilon_{*}^{s}$, is a relative injective resolution of $L_{*}$.

We may consider the differentials $\delta^{s}$ of this complex as the components of a single differential

$$
\delta^{*}: H L_{*, *}\left(L_{*}\right) \otimes \operatorname{gr}_{J}^{*}\left(R_{*}\right) \longrightarrow H L_{*-1, *}\left(L_{*}\right) \otimes \operatorname{gr}_{J}^{*+1}\left(R_{*}\right)
$$

on the $\operatorname{gr}_{J}^{*}\left(R_{*}\right)$-algebra $H L_{*, *}\left(L_{*}\right) \otimes \operatorname{gr}_{J}^{*}\left(R_{*}\right)$. By [36, Prop. 3.10] or 35, Prop. $7]$, it can be characterized as follows.

Proposition 4.7 The differential $\delta^{*}$ of (4.7) is a derivation over $\operatorname{gr}_{J}^{*}\left(R_{*}\right)$. As such, it is determined by its values $\delta^{*}\left(e_{j}\right)=-\left\{x_{j}\right\}$ on the generators $e_{j}$ of the algebra $H L_{*, *}\left(L_{*}\right)$.

Explicitly, for an element $e_{i_{1}} \wedge \cdots \wedge e_{i_{l}} \otimes \bar{f} \in H L_{*, *}\left(L_{*}\right) \otimes \operatorname{gr}_{J}^{*}\left(R_{*}\right)$, we have

$$
\delta^{*}\left(e_{i_{1}} \wedge \cdots \wedge e_{i_{l}} \otimes \bar{f}\right)=\sum_{j=1}^{l}(-1)^{j+1} e_{i_{1}} \wedge \cdots \wedge \widehat{e}_{i_{j}} \wedge \cdots \wedge e_{i_{l}} \otimes \overline{x_{i_{j}} f},
$$

where the hat indicates that the entry underneath is omitted. 


\section{Adams resolutions}

In this section, we recall the notion of Adams resolutions with respect to a given injective class in a triangulated category. We then indicate how the injective classes giving rise to the traditional Adams resolutions can be obtained in a natural way. In an analogous way, we obtain the injective class we need for the construction of the $I$-adic tower. We are rather brief here. A more detailed exposition can be found in [9, see also [10] and [18. The original reference for Adams resolutions (in the classical sense) is [26].

A length two complex in an additive category $\mathcal{C}$ is a diagram

$$
F \stackrel{f}{\longrightarrow} L \stackrel{g}{\longrightarrow} M
$$

with $g f=0$. A complex is a sequence

$$
\cdots \longrightarrow M_{s+1} \stackrel{f_{s+1}}{\longrightarrow} M_{s} \stackrel{f_{s}}{\longrightarrow} M_{s+1} \longrightarrow \cdots
$$

such that $f_{s} f_{s+1}=0$ for all $s$. If the terms $M_{s}$ of a complex are zero for all large $s$ or all small $s$, we omit them from the notation. An injective class $\mathcal{J}$ in $\mathcal{C}$ is specified by a collection of length two complexes, the so-called allowable exact complexes, and a collection of objects called the injectives. The two collections are required to determine each other in the following way. An object $J$ is injective if and only if the sequence

$$
[M, J]^{*} \stackrel{g^{*}}{\longrightarrow}[L, J]^{*} \stackrel{f^{*}}{\longrightarrow}[F, J]^{*}
$$

of graded abelian groups is exact for each allowable exact complex of the form (5.1), and vice versa. Furthermore, one requires that there are enough injectives, in the following sense. For each map $f: M \rightarrow N$ there exists an injective $I$ and a map $g: N \rightarrow I$ such that $M \stackrel{f}{\rightarrow} N \stackrel{g}{\rightarrow} I$ is an allowable exact complex.

Now assume that $\mathcal{C}$ is triangulated. Then we may specify instead of the allowable exact complexes a family of morphisms called the null-maps. An object $J$ is then injective if and only if the homomorphism of graded abelian groups

$$
[N, J]^{*} \stackrel{f^{*}}{\longrightarrow}[M, J]^{*}
$$

is zero for each null map $f: M \rightarrow N$, and vice versa.

An example for an injective class is the split injective class, defined in each additive category $\mathcal{C}$. Let $\vee$ denote the biproduct (coproduct and product) in $\mathcal{C}$. The allowable complexes are the ones of the form

$$
A \vee B \longrightarrow B \vee C \longrightarrow C \vee D
$$


where the maps are the projections onto the second summand followed by the inclusions into the first summand. Every object is injective with respect to this injective class. If $\mathcal{C}$ is triangulated, the null-maps are the trivial maps.

There is the dual concept of a projective class in an additive category $\mathcal{C}$. It is specified by a collection of length two complexes called the allowable exact complexes, and a collection of objects called the projectives. An object $P$ is projective if and only if

$$
[P, F]^{*} \stackrel{f_{*}}{\longrightarrow}[P, L]^{*} \stackrel{g_{*}}{\longrightarrow}[P, M]^{*}
$$

is exact for each allowable exact complex of the form (5.1), and vice versa. There have to exist enough projectives, in an obvious sense. If $\mathcal{C}$ is triangulated, we can specify instead of the allowable exact sequences the collection of null-maps. They are all the maps $f: M \rightarrow N$ for which

$$
f_{*}:[P, M]^{*} \longrightarrow[P, N]^{*}
$$

is trivial for each projective $P$, and vice versa.

Suppose that $\mathcal{J}$ is an injective class in an additive category $\mathcal{C}$. A relative injective resolution of an object $M$ with respect to $\mathcal{J}$ is a complex of the form

$$
0 \longrightarrow M \longrightarrow J_{0} \longrightarrow J_{1} \longrightarrow J_{2} \longrightarrow \cdots
$$

with injectives $J_{i}$ which has the property that each three-term subcomplex

$$
J_{s-1} \longrightarrow J_{s} \longrightarrow J_{s+1},
$$

where $J_{-1}=M$ and $J_{s}=0$ for $s<-1$, is allowable exact. If $\mathcal{C}$ is triangulated, such a resolution gives rise to an Adams resolution of $M$ with respect to $\mathcal{J}$, which is unique up to isomorphism. By this, we mean a diagram of the form

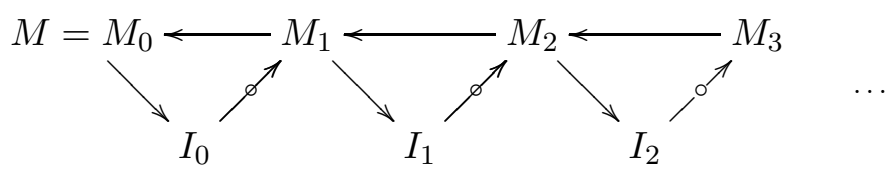

consisting of triangles

$$
M_{i+1} \longrightarrow M_{i} \longrightarrow I_{i} \longrightarrow \Sigma M_{i+1}
$$

such that all $I_{i}$ are injectives and all maps $M_{i+1} \rightarrow M_{i}$ are null-maps. Here $\Sigma$ denotes the suspension of $\mathcal{C}$. The injectives that we get from a relative injective resolution (5.2) are $I_{i}=\Sigma^{-i} J_{i}$. Conversely, we can derive from an Adams resolution a relative injective resolution. Applying $[N,-]^{*}$ to an Adams resolution, where $N$ is another object of $\mathcal{C}$, yields an exact couple of abelian groups. It gives rise to an Adams spectral sequence. 
Dually defined are relative projective resolutions and Adams resolutions with respect to a projective class.

There is a standard method for transferring an injective class from one additive category to another, namely by means of an adjunction. Let $\mathcal{C}$ and $\mathcal{C}^{\prime}$ be additive categories. Assume that we have an adjoint pair of additive functors

$$
\mathcal{C} \underset{G}{\stackrel{F}{\rightleftarrows}} \mathcal{C}^{\prime}
$$

where $F$ is the left adjoint. Given an injective class $\mathcal{J}^{\prime}$ in $\mathcal{C}^{\prime}$, we define an injective class $F^{-1}\left(\mathcal{J}^{\prime}\right)$ in $\mathcal{C}$ as follows. The allowable exact complexes are all length two complexes whose image under $F$ is allowable exact in $\mathcal{C}^{\prime}$. The associated injectives turn out to be the retracts of objects of the form $G(X)$, where $X$ is a $\mathcal{J}^{\prime}$-injective.

The injective classes in the stable homotopy category $\mathscr{D}_{\mathbb{S}}$ which give rise to classical Adams resolutions arise in this way. Namely, for a ring spectrum $R$, we have an adjoint pair of additive functors

$$
\mathscr{D}_{\mathbb{S}} \underset{U=F_{\mathbb{S}}(R,-)}{\stackrel{V=R \mathbb{S}^{-}}{\underset{\mathrm{N}}{\rightleftarrows}}} \mathscr{D}_{\mathbb{S}}
$$

The Adams injective class with respect to $R$ is $V^{-1}(\mathcal{J})$, where $\mathcal{J}$ is the split injective class in $\mathscr{D}_{\mathbb{S}}$. Its injectives are the retracts of spectra of the form $F_{\mathbb{S}}(R, X)$. These agree with the retracts of spectra of the form $R \wedge_{\mathbb{S}} X$ (see Remark 5.2). More generally, if $F$ is a ring spectrum over a commutative $\mathbb{S}$-algebra $R$, we have an additive adjunction

$$
\mathscr{D}_{R} \underset{U=F_{R}(F,-)}{\stackrel{V=F \wedge-}{\rightleftarrows}} \mathscr{D}_{R}
$$

Definition 5.1 Let $\mathcal{J}$ be the split injective class in $\mathscr{D}_{R}$. The Adams injective class associated to $F$ in $\mathscr{D}_{R}$ is the injective class given via (5.3) as $V^{-1}(\mathcal{J})$.

Similarly to the situation in $\mathscr{D}_{\mathbb{S}}$, the injectives are the retracts of $R$-modules of the form $F \wedge M$, where $M$ is an $R$-module.

Remark 5.2 Note that we have an adjunction (5.3) for any object $F$ in $\mathscr{D}_{R}$, not just for ring spectra. It is the recognition of the injectives mentioned above which depends on having a multiplicative structure on $F$. Indeed, this implies that $V$ is a monad and $U$ a comonad, and as $V$ and $U$ are adjoint, the $V-$ algebras (the retracts of $F \wedge M, M \in \mathscr{D}_{R}$ ) are the same as the $U$-coalgebras (the retracts of $\left.F_{R}(F, M), M \in \mathscr{D}_{R}\right)$. 
Remark 5.3 In a completely analogous way, one can define an Adams injective class associated to a monoid $F_{*}$ in the derived category $\mathscr{D}_{R_{*}}$ of a graded ring $R_{*}$. In particular, this applies to an algebra $F_{*}$ over $R_{*}$ or more generally to a differential graded algebra $F_{*}$. Instead of (15.3), one uses the adjunction

$$
\mathscr{D}_{R_{*}} \underset{U=\mathbf{R H o m}\left(F_{*},-\right)}{\stackrel{V=F_{*} \otimes^{\mathbf{L}}-}{\rightleftarrows}} \mathscr{D}_{R_{*}}
$$

(see Section 4 for the definition of $\otimes^{\mathbf{L}}$ and $\mathbf{R H o m}$ ).

We also mention the ghost projective class. It is defined in the triangulated categories $\mathscr{D}_{R}$, where $R$ is an $\mathbb{S}$-algebra. The null-maps are the morphisms which are trivial on homotopy groups. The projectives are the retracts of wedges of suspensions of $R$.

\section{Construction of the tower}

Let $(R, T, F)$ be a regular triple with $F \cong T \wedge L$. Thus we have $L_{*} \cong R_{*} / J$ and $F_{*} \cong T_{*} / I$, where $J$ is generated by a sequence $S=\left(x_{0}, x_{1}, \ldots\right) \subseteq R_{*}$, regular on $R_{*}$ and on $T_{*}$, and where $I=J \cdot T_{*}$. Consider the graded polynomial ring $L_{*}\left[y_{0}, y_{1}, \ldots\right]$ with $\left|y_{j}\right|=\left|x_{j}\right|$. Define $L$-free $R$-modules $J^{s} / J^{s+1}$ by setting

$$
J^{s} / J^{s+1}=\bigvee_{y \in V_{s}} \Sigma^{|y|} L
$$

where $V_{s}$ is the set of monomials of homogeneous degree $s$ in the variables $y_{j}$. By " $L$-free", we mean isomorphic to a wedge of suspensions of copies of $L$.

We now construct a sequence in $\mathscr{D}_{R}$ of the form:

$$
* \longrightarrow R \stackrel{\eta}{\longrightarrow} L \stackrel{\widetilde{\varepsilon}^{0}}{\longrightarrow} \Sigma J / J^{2} \stackrel{\Sigma \widetilde{\varepsilon}^{1}}{\longrightarrow} \Sigma^{2} J^{2} / J^{3} \stackrel{\Sigma^{2} \widetilde{\varepsilon}^{2}}{\longrightarrow} \cdots
$$

The maps $\widetilde{\varepsilon}^{i}$ are easy to describe. Namely, as all terms $J^{s} / J^{s+1}$ are $L$-free, a map between them corresponds to a matrix over the graded endomorphism ring $L_{R}^{*}(L)$. The Bockstein operations define a map

$$
\widetilde{\varepsilon}^{0}=\bigvee_{j} Q_{j}: L \longrightarrow \Sigma J / J^{2}
$$

For $s>0$, we define $\widetilde{\varepsilon}^{s}: J^{s} / J^{s+1} \longrightarrow \Sigma J^{s+1} / J^{s+2}$ to be the sum over all monomials $y \in V_{s}$ of the maps

$$
\bigvee_{j} \Sigma^{|y|} Q_{j}: \Sigma^{|y|} L \longrightarrow \bigvee_{j} \Sigma^{\left|y y_{j}\right|+1} L
$$

Recall from Proposition 3.5 that $L_{*}^{R}(L) \cong \Lambda_{L_{*}}\left(a_{0}, a_{1}, \ldots\right)$ as an $L_{*}$-module. 
Lemma 6.1 The L-homology of the sequence

$$
* \longrightarrow R \stackrel{\eta}{\longrightarrow} L \stackrel{\widetilde{\varepsilon}^{0}}{\longrightarrow} \Sigma J / J^{2} \stackrel{\Sigma \widetilde{\varepsilon}^{1}}{\longrightarrow} \Sigma^{2} J^{2} / J^{3} \stackrel{\Sigma^{2} \widetilde{\varepsilon}^{2}}{\longrightarrow} \cdots
$$

is a long exact sequence of $\Lambda_{L_{*}}\left(a_{0}, a_{1}, \ldots\right)$-comodules

$$
0 \longrightarrow L_{*} \stackrel{\eta_{*}}{\longrightarrow} L_{*}^{R}(L) \stackrel{\widetilde{\varepsilon}_{*}^{0}}{\longrightarrow} L_{*}^{R}\left(\Sigma J / J^{2}\right) \stackrel{\widetilde{\varepsilon}_{*}^{1}}{\longrightarrow} L_{*}^{R}\left(\Sigma J^{2} / J^{3}\right) \longrightarrow \cdots
$$

which provides a relative injective resolution of $L_{*}$.

Proof The isomorphism $L_{*}^{R}(L) \cong \Lambda_{L_{*}}\left(a_{0}, a_{1}, \ldots\right)$ from Proposition 3.5 induces

$$
L_{*}^{R}\left(J^{s} / J^{s+1}\right) \cong L_{*}^{R}(L) \otimes_{L_{*}} J^{s} / J^{s+1} .
$$

We have identified in Lemma 3.16 the morphism $L_{*}^{R}\left(Q_{j}\right)$. Recall that we have obtained in Corollary 4.6 and Proposition 4.7 a relative injective resolution of $L_{*}$ over $\Lambda_{L_{*}}\left(e_{0}, e_{1}, \ldots\right)$. It follows from the definition of the maps $\widetilde{\varepsilon}^{s}$ that mapping the generators $a_{j}$ of $\Lambda_{L_{*}}\left(a_{0}, a_{1}, \ldots\right)$ to the generators $e_{j}$ yields an isomorphism between (6.2) and the sequence in Corollary 4.6. if we endow the latter with the total gradation.

From now on, we will omit the index $s$ and just write $\widetilde{\varepsilon}$ for all the maps in the sequence. To obtain an abstract characterization of the sequence (6.1) by means of Lemma 6.1, we need the following technical lemma. Recall from Section 1 the difference between the concepts of a module spectrum over a ring spectrum and a module over an $\mathbb{S}$-algebra. We write Cohom* for the maps of graded comodules over some graded coalgebra.

Lemma 6.2 Let $E$ be an arbitrary $R$-ring spectrum. Let $Y$ be an $R$-module for which $E_{*}^{R}(Y)$ is $E_{*}$-free and let $Z$ be an $E$-module spectrum in $\mathscr{D}_{R}$. Then there is a natural isomorphism of $E_{*}$-modules

$$
[Y, Z]_{R}^{*} \cong \operatorname{Hom}_{E_{*}}^{*}\left(E_{*}^{R}(Y), Z_{*}\right) .
$$

If $E$ is a commutative $R$-ring spectrum and $E_{*}^{R}(E)$ is $E_{*}$-flat, the functor $E_{*}^{R}(-)$ induces an isomorphism of $E_{*}$-modules

$$
[Y, Z]_{R}^{*} \cong \operatorname{Cohom}_{E_{*}^{R}(E)}^{*}\left(E_{*}^{R}(Y), E_{*}^{R}(Z)\right) .
$$

Proof First, we construct a natural transformation of functors of $E$-module spectra with values in $E_{*}$-modules

$$
[Y,-]_{R}^{*} \longrightarrow \operatorname{Hom}_{E_{*}}^{*}\left(E_{*}^{R}(Y),(-)_{*}\right)
$$


as follows. The $E$-homology functor gives rise to a natural transformation

$$
[Y,-]_{R}^{*} \longrightarrow \operatorname{Hom}_{E_{*}}^{*}\left(E_{*}^{R}(Y), E_{*}^{R}(-)\right) .
$$

Composing this with the transformation $E_{*}^{R}(-) \rightarrow(-)_{*}$ induced by the action map for $E$-module spectra induces a transformation as in (6.3).

If we precompose both sides of (6.3) with $E \wedge_{R}-$, we obtain a transformation

$$
[Y, E \wedge-]_{R}^{*} \longrightarrow \operatorname{Hom}_{E_{*}}^{*}\left(E_{*}^{R}(Y), E_{*}^{R}(-)\right)
$$

of functors of $R$-modules. As $E_{*}^{R}(Y)$ is $E_{*}$-free, the functor on the right hand side is exact. Hence both sides of (6.5) define homology theories on $\mathscr{D}_{R}$. On suspensions of $R$, (6.5) is the duality morphism (2.2), and is therefore an isomorphism. Hence it is an equivalence on $\mathscr{D}_{R}$ by the usual argument. So (6.3) is an equivalence on all $E$-module spectra $Z$ of the form $E \wedge X, X$ an $R$ module. But an arbitrary $E$-module spectrum $Z$ in $\mathscr{D}_{R}$ is a retract of $E \wedge Z$, via the action map. So we obtain an isomorphism for general $Z$ by naturality.

For commutative $E$ for which $E_{*}^{R}(E)$ is $E_{*}$-flat, $E_{*}^{R}(-)$ takes values in $E_{*}^{R}(E)-$ comodules. The structure maps are defined exactly as in the case $R=S$. Consider the composition of natural transformations of functors of $E$-module spectra

$$
E_{*}^{R}(E) \otimes(-)_{*} \stackrel{E_{*}^{R}(E) \otimes \eta_{*}}{\longrightarrow} E_{*}^{R}(E) \otimes E_{*}^{R}(-) \stackrel{\times}{\longrightarrow} E_{*}^{R}(E \wedge-) \longrightarrow E_{*}^{R}(-),
$$

where the last morphism is induced by the $E$-action. It can be checked to be $E_{*}^{R}(E)$-colinear. Precomposing it with $E \wedge-$ yields a transformation

$$
E_{*}^{R}(E) \otimes E_{*}^{R}(-) \longrightarrow E_{*}^{R}(E \wedge-)
$$

of homology theories on $\mathscr{D}_{R}$. It does not agree with the one induced by the exterior product $\times$. Nevertheless, an inspection shows that it defines an equivalence of homology theories. With an argument as above, we conclude that (6.6) is an equivalence. Now recall that we have an adjunction between $E_{*}^{R}(E)$-comodules and $E_{*}$-modules

$$
\operatorname{Mod}_{E_{*}} \stackrel{E_{*}^{R}(E) \otimes-}{\longleftarrow} \operatorname{Comod}_{E_{*}^{R}(E)},
$$

where $U$ is the forgetful functor. Thus we obtain

$$
\operatorname{Cohom}_{E_{*}^{R}(E)}^{*}\left(E_{*}^{R}(Y), E_{*}^{R}(Z)\right) \cong \operatorname{Hom}_{E_{*}}^{*}\left(E_{*}^{R}(Y), Z_{*}\right),
$$

and the second statement follows from the first. 
We are now in a position to construct the $I$-adic tower. To identify its homotopy and $F$-homology groups, however, we need a few facts concerning universal coefficient and Künneth spectral sequences. We recall these first.

Let $M$ and $N$ be $R$-modules. Then we have [11, IV.4] a universal coefficient spectral sequence of the form

$$
E_{2}^{*, *}=\operatorname{Ext}_{R^{*}}^{*, *}\left(M^{*}, N^{*}\right) \quad \Longrightarrow \quad N_{R}^{*}(M) .
$$

It is a conditionally convergent half-plane spectral sequence with entering differentials, in the terminology of Boardman [7]. If $M=N$, it is multiplicative with respect to composition. It is shown in [9, §7.3] that this spectral sequences is in fact an Adams spectral sequence, with respect to the ghost projective class in $\mathscr{D}_{R}$.

Let us recall what we can say about the detection of a given non-trivial map $f: M \rightarrow N$. If the induced map $f_{*}: M_{*} \rightarrow N_{*}$ on homotopy groups is nontrivial, $f$ is represented in (6.7) by $f_{*}$. This follows from the construction of the spectral sequence. Assume now that $f_{*}$ is zero. Denoting the homotopy cofibre of $f$ by $C$, we then have a short exact sequence of $R_{*}$-modules

$$
0 \longrightarrow N_{*} \longrightarrow C_{*} \longrightarrow M_{*-1} \longrightarrow 0 .
$$

Let $t$ be the element of $\operatorname{Ext}_{R_{*}}^{1,-1}\left(M_{*}, N_{*}\right)$ representing it. We leave the verification of the following fact to the reader.

Proposition 6.3 If the element $t$ is non-trivial, it represents the map $f$ in the $E_{2}$-term of (6.7). Otherwise, $f$ has filtration degree strictly greater than one.

Let $P$ be a further $R$-module. There is [11, IV.4] a Künneth spectral sequence of the form

$$
E_{*, *}^{2}=\operatorname{Tor}_{*, *}^{R_{*}}\left(P_{*}, M_{*}\right) \quad \Longrightarrow \quad P_{*}^{R}(M) .
$$

It is a strongly convergent half-plane spectral sequence with exiting differentials.

We will need a statement about naturality. Namely, let $f: M \rightarrow N$ be a nontrivial map inducing zero on homotopy groups, as above. Consider the Künneth spectral sequences (6.9) and

$$
\bar{E}_{*, *}^{2}=\operatorname{Tor}_{*, *}^{R_{*}}\left(P_{*}, N_{*}\right) \quad \Longrightarrow \quad P_{*}^{R}(N) .
$$

As $f_{*}: M_{*} \rightarrow N_{*}$ is zero, the map $f_{*}^{r}: E_{*, *}^{r} \rightarrow \bar{E}_{*, *}^{r}$ induced by $f$ on the spectral sequences is zero. Because the spectral sequences converge strongly, 
this implies that $P_{*}^{R}(f): P_{*}^{R}(M) \rightarrow P_{*}^{R}(N)$ induces zero on the associated graded. Therefore, $P_{*}^{R}(f)$ induces a map of degree 1

$$
\operatorname{gr}^{*}\left(P_{*}^{R}(M)\right) \longrightarrow \operatorname{gr}^{*+1}\left(P_{*}^{R}(N)\right) .
$$

Similarly, the morphism $P_{R}^{*}(f): P_{R}^{*}(N) \rightarrow P_{R}^{*}(M)$ induces a map

$$
\operatorname{gr}^{*}\left(P_{R}^{*}(N)\right) \longrightarrow \operatorname{gr}^{*+1}\left(P_{R}^{*}(M)\right) .
$$

Indeed, to deduce that $P_{R}^{*}(f)$ induces zero on the associated graded, it suffices to know that the respective spectral sequences are conditionally convergent, see [7. Lemma 5.6]. The following statement is proved in a similar way as the Geometric Boundary Theorem [28, Thm. 2.3.4].

Proposition 6.4 The homomorphism (6.11) is represented on the Künneth spectral sequences (6.9) and (6.10) by a morphism $E_{*, *}^{r} \rightarrow \bar{E}_{*-1, *}^{r}$ of degree -1 . On $E^{2}$-terms, it is given by the connecting homomorphism

$$
\operatorname{Tor}_{*, *}^{R_{*}}\left(P_{*}, M_{*}\right) \stackrel{\partial}{\longrightarrow} \operatorname{Tor}_{*-1, *}^{R_{*}}\left(P_{*}, N_{*}\right)
$$

associated to the short exact sequence (6.8). A similar statement holds for the homomorphism (6.12) and the universal coefficient spectral sequences converging to $P_{R}^{*}(N)$ and $P_{R}^{*}(M)$.

Now consider the universal coefficient spectral sequence

$$
E_{2}^{*, *}=\operatorname{Ext}_{R^{*}}^{* *}\left(L^{*}, L^{*}\right) \Longrightarrow L_{R}^{*}(L) .
$$

By Proposition 4.2, we have an isomorphism of $L_{*}$-algebras

$$
\operatorname{Ext}_{R^{*}}^{*, *}\left(L^{*}, L^{*}\right) \cong \widehat{\Lambda}_{L^{*}}\left(f_{0}, f_{1}, \ldots\right) ;
$$

the $f_{j}$ were explicitly defined there. We already know from Proposition 3.5 that there is an isomorphism of $L_{*}$-algebras

$$
L_{R}^{*}(L) \cong \widehat{\Lambda}_{L_{*}}\left(Q_{0}, Q_{1}, \ldots\right) .
$$

Proposition 6.5 The Bockstein operation $Q_{j}: L \rightarrow \Sigma^{\left|x_{j}\right|+1} L$ is represented in the spectral sequence (6.13) by the element $f_{j}$. Therefore, the spectral sequence collapses.

Proof Consider the cofibre sequence in $\mathscr{D}_{R}$ associated to $Q_{j}$

$$
L \stackrel{Q_{j}}{\longrightarrow} \Sigma^{\left|x_{j}\right|+1} L \longrightarrow C_{j} \longrightarrow \Sigma L .
$$


By Proposition 6.3. it suffices to verify two things. Firstly, that $Q_{j}$ is trivial on homotopy groups. Secondly, that the thereby obtained short exact sequence

$$
0 \longrightarrow L_{*-\left|x_{j}\right|-1} \longrightarrow\left(C_{j}\right)_{*} \longrightarrow L_{*-1} \longrightarrow 0
$$

corresponds to $f_{j}$ in $\operatorname{Ext}_{R_{*}}^{1, *}\left(L_{*}, L_{*}\right)$. For the first statement, note that the morphism induced by $Q_{j}$ on homotopy groups is trivial for degree reasons.

For the second statement, recall that $Q_{j}$ is obtained by smashing the composition

$$
\bar{\beta}_{j}: R / x_{j} \stackrel{\beta_{j}}{\longrightarrow} \Sigma^{\left|x_{j}\right|+1} R \stackrel{\rho_{j}}{\longrightarrow} \Sigma^{\left|x_{j}\right|+1} R / x_{j}
$$

with $\bigwedge_{i \neq j} R / x_{i}$. Applying the octahedral axiom (see [15. Appendix A] for instance) to the induced decomposition of $Q_{j}$, we find that (6.15) is isomorphic to:

$$
0 \longrightarrow \Sigma^{\left|x_{j}\right|+1} L_{*} \stackrel{x_{j}}{\longrightarrow} X_{*}=R_{*} /\left(x_{j}^{2}, x_{i} ; i \neq j\right) \longrightarrow L_{*} \longrightarrow 0
$$

Now the statement follows from the fact that the element $f_{j} \in \operatorname{Ext}_{R_{*}}^{1, *}\left(L_{*}, L_{*}\right)$ corresponds to (6.16). This verification is left to the reader.

We are now ready to prove our main statement. Note the special case $T=R$.

Theorem 6.6 Let $(R, T, F)$ be a regular triple with $F=T \wedge L$.

(i) Applying $T \wedge-$ to the sequence (6.1) yields a relative injective resolution

$$
* \longrightarrow T \longrightarrow F \longrightarrow \Sigma I / I^{2} \longrightarrow \Sigma^{2} I^{2} / I^{3} \longrightarrow \cdots
$$

of $T$ with respect to the Adams injective class associated to $L$ in $\mathscr{D}_{R}$ and hence with respect to the one associated to $F=T \wedge L$.

(ii) The Adams resolution associated to (6.17), a diagram of $T$-module spectra, is of the form:

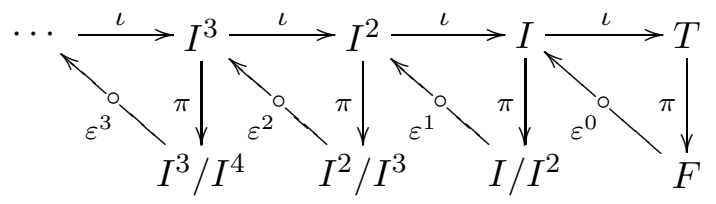

The induced diagram of homotopy groups is isomorphic to

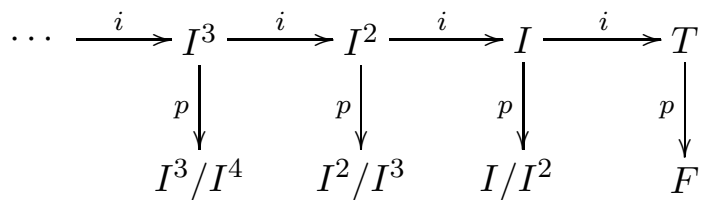


composed of short exact sequences. The induced diagram of $F$-homology groups is isomorphic to the image of the diagram (4.4) under the functor $F_{*}^{R}(T) \otimes-$, equipped with the total gradation.

Definition 6.7 We call (6.18) the topological I-adic tower over $T$.

Proof (i) First, we verify that (6.1) is a relative injective resolution of $R$ with respect to $L$. Clearly, all objects in the sequence (6.1), except for $R$, are $L$-injective. Let $N$ be an arbitrary $R$-module. By definition of the Adams injective class associated to $F$ in $\mathscr{D}_{R}$, we have to check that the sequence

$$
\cdots \longrightarrow\left[L \wedge J^{s+1} / J^{s+2}, N\right]_{R}^{*-1} \stackrel{\left(\widetilde{\varepsilon}^{s}\right)^{*}}{\longrightarrow}\left[L \wedge J^{s} / J^{s+1}, N\right]_{R}^{*} \longrightarrow \cdots
$$

obtained by applying the functor $[L \wedge-, N]_{R}^{*}$ to (6.1) is exact. The adjunction (5.3) (for $F=L$ ) and Lemma 6.2 imply

$$
\left[L \wedge J^{s} / J^{s+1}, N\right]_{R}^{*} \cong\left[J^{s} / J^{s+1}, L_{R}(L, N)\right]_{R}^{*} \cong\left(L_{*}^{R}\left(J^{s} / J^{s+1}\right), N_{R}^{-*}(L)\right)_{L_{*}}^{*}
$$

where $(-,-)_{L_{*}}^{*}=\operatorname{Hom}_{L_{*}}^{*}(-,-)$. But by Lemma 6.1 the $L$-homology of the sequence (6.1) splits into short exact sequences of $L_{*}$-modules. It follows that (6.20) is exact. Now clearly (6.17) is an $L$-resolution of $T$. As $F$ is of the form $T \wedge L$, it is also an $F$-resolution.

(ii) Consider the Adams resolution associated to the sequence (6.1) first. It is of the form:

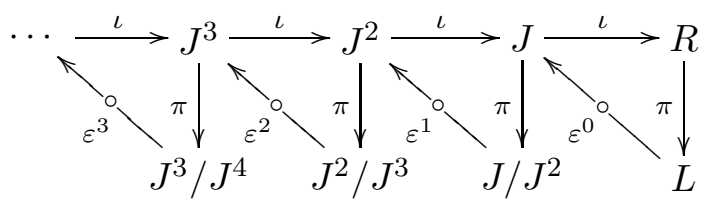

We claim that the diagram of homotopy groups of the tower is isomorphic to the diagram of homology groups of (4.1), i.e. to:

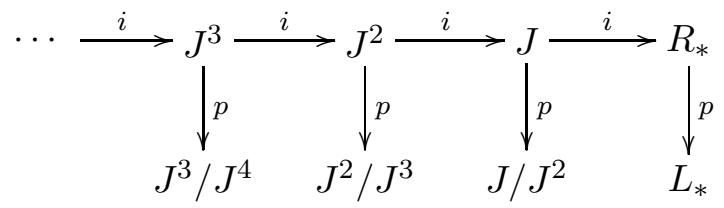

It is clear that the sequence of homotopy groups of the triangle

$$
J \stackrel{i}{\longrightarrow} R \stackrel{\pi}{\longrightarrow} L \stackrel{\varepsilon^{0}}{\longrightarrow} \Sigma J
$$

splits into the short exact sequence

$$
\mathcal{E}^{0}: \quad 0 \longrightarrow J \stackrel{i}{\longrightarrow} R_{*} \stackrel{p}{\longrightarrow} L_{*} \longrightarrow 0 .
$$


Consider the commutative diagram:

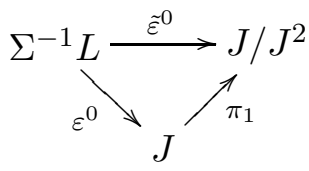

Here, $\pi_{1}$ is the unique lift of $\tilde{\varepsilon}^{0}$ to $J$. It is unique because $J$ is the first term of the uniquely determined Adams resolution associated to (6.1). We need to show that it induces the canonical projection $p_{1}$ on coefficients. For this, we consider the pair of universal coefficient spectral sequences:

$$
\begin{gathered}
E_{2}^{*, *}=\operatorname{Ext}_{R_{*}^{* *}}^{*}\left(J, J / J^{2}\right) \Longrightarrow\left[J, J / J^{2}\right]_{R}^{*} \\
\bar{E}_{2}^{*+1, *}=\operatorname{Ext}_{R^{*}}^{*+1, *}\left(L_{*}, J / J^{2}\right) \Longrightarrow\left[L, J / J^{2}\right]_{R}^{*+1}
\end{gathered}
$$

By Proposition 6.4 the morphism $\left[J, J / J^{2}\right]_{R}^{*} \stackrel{\left(\varepsilon^{0}\right)^{*}}{\longrightarrow}\left[L, J / J^{2}\right]_{R}^{*+1}$ induced by $\varepsilon^{0}$ is represented by a morphism of degree one on the spectral sequences. On $E_{2}$-terms it is given by the connecting homomorphism

$$
\varepsilon^{0}: \operatorname{Ext}_{R^{*}}^{*, *}\left(J, J / J^{2}\right) \longrightarrow \operatorname{Ext}_{R^{*}}^{*+1, *}\left(L_{*}, J / J^{2}\right)
$$

associated to $\mathcal{E}^{0}$. Let $x_{1} \in E_{2}^{*, *}$ and $y_{0} \in \bar{E}_{2}^{*, *}$ be representatives of $\pi_{1}$ and $\tilde{\varepsilon}^{0}$ respectively. We need to check that $x_{1}=p_{1}$. As $\tilde{\varepsilon}^{0}=\left(\varepsilon^{0}\right)^{*}\left(\pi_{1}\right)$, we have $y_{0}=\varepsilon^{0}\left(x_{1}\right)$ in case that $\varepsilon^{0}\left(x_{1}\right)$ is non-trivial. However, it follows from Theorem 4.3 and Remark 4.5 that $\varepsilon^{0}$ is monomorphic. So it suffices to identify $y_{0}$ and to show that $\varepsilon^{0}\left(p_{1}\right)=y_{0}$. Proposition 6.5] implies that

$$
\widetilde{\varepsilon}^{0}=\bigvee_{j} Q_{j}: L \rightarrow \Sigma J / J^{2}
$$

is represented by

$$
y_{0}=\sum f_{j} \otimes\left\{x_{j}\right\} \in \operatorname{Ext}_{R_{*}}^{1, *}\left(L_{*}, L_{*}\right) \otimes_{L^{*}} J / J^{2} \cong \operatorname{Ext}_{R_{*}}^{1, *}\left(L_{*}, J / J^{2}\right) .
$$

The $f_{j}$ were defined in such a way that $y_{0}$ corresponds to the short exact sequence

$$
\mathcal{F}^{1}: \quad 0 \longrightarrow J / J^{2} \stackrel{j_{1}}{\longrightarrow} R_{*} / J^{2} \stackrel{q_{2}}{\longrightarrow} L_{*} \longrightarrow 0 .
$$

It remains to verify that $\varepsilon^{0}\left(p_{1}\right)$ is represented by $\mathcal{F}^{1}$. By definition, the connecting homomorphism $\varepsilon^{0}$ maps the homomorphism $p_{1}: J \rightarrow J / J^{2}$ to the pushout of the short exact sequence $\mathcal{E}^{0}$ along $p_{1}$. This pushout is $\mathcal{F}^{1}$, as the diagram of short exact sequences

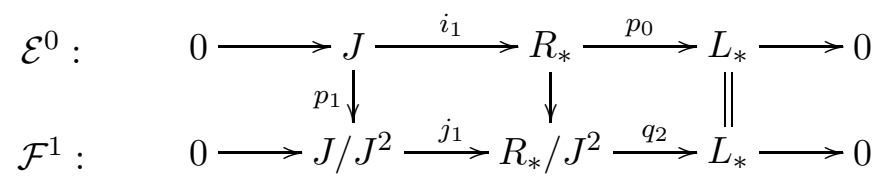


shows. Here, the middle vertical map is the projection. We conclude that

$$
J^{2} \stackrel{\iota}{\longrightarrow} J \stackrel{\pi}{\longrightarrow} J / J^{2} \stackrel{\varepsilon^{1}}{\longrightarrow} \Sigma J^{2}
$$

realizes the extension $\mathcal{E}^{1}$. We can carry on by induction.

Let us now compute the homotopy groups of the Adams resolution (6.18) associated to (6.17). If we smash (6.21) with $T$, we obtain another tower which qualifies as an Adams resolution associated to (6.17). By uniqueness of Adams resolutions, the two towers must be isomorphic. The product on $T_{*}$ and the natural transformation $T_{*} \otimes_{R_{*}}-\rightarrow(T \wedge-)_{*}$ induce a commutative diagram

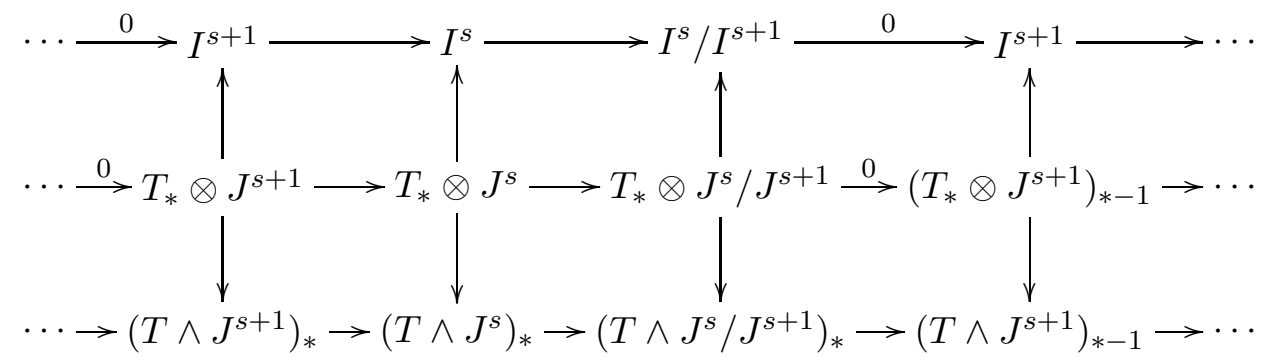

where $\otimes=\otimes_{R_{*}}$. We show that all vertical maps are isomorphisms. For the upper row it suffices to note that

$$
\operatorname{Tor}_{k}^{R_{*}}\left(T_{*}, J^{s} / J^{s+1}\right)=\operatorname{Tor}_{k}^{R_{*}}\left(T_{*}, R_{*} / J\right) \otimes_{L_{*}} J^{s} / J^{s+1}=0
$$

for $k \neq 0$, which is a consequence of the assumption that the sequence generating the ideal $J$ is regular on $T_{*}$. By construction of $J^{s} / J^{s+1}$, we have

$$
\left(T \wedge J^{s} / J^{s+1}\right)_{*} \cong I^{s} / I^{s+1}
$$

Using this fact and starting with $\left(T \wedge J^{0}\right)_{*}=(T \wedge R)_{*} \cong T_{*}$, we show inductively that $\left(T \wedge J^{s}\right)_{*} \cong I^{s}$, using the Five-Lemma. We conclude that the homotopy groups are as asserted.

The identification of the $L$-homology is easier. We show first that $L_{*}^{R}(-)$ of the $J$-adic tower (6.21) is isomorphic to (4.4), endowed with the total gradation. In the proof of Lemma 6.1 we have used the fact that $L_{*}^{R}(-)$ of the sequence (6.1) is isomorphic to the long exact sequence obtained from Theorem 4.3

$$
0 \rightarrow L_{*} \stackrel{p_{*}}{\longrightarrow} H L_{*, *}\left(L_{*}\right) \stackrel{\delta^{0}}{\longrightarrow} H L_{*-1, *}\left(J / J^{2}\right) \stackrel{\delta^{1}}{\longrightarrow} H L_{*-2, *}\left(J^{2} / J^{3}\right) \rightarrow \cdots
$$

endowed with the total gradation. It follows that we have isomorphisms of $L_{*}$-modules:

$$
L_{*-1}^{R}(J) \cong \operatorname{coker}\left(\eta_{*}: L_{*} \stackrel{\pi}{\rightarrow} L_{*}^{R}(L)\right) \cong \operatorname{coker}\left(p_{*}\right) \cong H L_{*-1, *}(J)
$$


Furthermore, the morphism $\left(\pi_{1}\right)_{*}: L_{*}^{R}(J) \rightarrow L_{*}^{R}\left(J / J^{2}\right)$ is injective, as it corresponds to $\left(p_{1}\right)_{*}: H L_{*, *}(J) \rightarrow H L_{*, *}\left(J / J^{2}\right)$. The fact that (6.23) is a cofibration implies that $L_{*}^{R}\left(\Sigma J^{2}\right) \cong \operatorname{coker}\left(\pi_{1}\right)_{*}$. By an inductive argument, the statement follows. In particular, we obtain that all $L$-homology groups are $L_{*}$-free and hence that all $F$-homology groups are $F_{*}$-free. Therefore Proposition $2.1 \mathrm{im}$ plies that we have Künneth isomorphisms

$$
F_{*}^{R}(T \wedge X) \cong F_{*}^{R}(T) \otimes F_{*}^{R}(X)
$$

for all terms $X$ in the $J$-adic tower. Thus we find that the $F$-homology of the $I$-adic tower (6.18) is as claimed.

Remark 6.8 Consider the sequence in $\mathscr{D}_{R_{*}}$

$$
T_{*} \longrightarrow F_{*} \longrightarrow \mathrm{\circ} \longrightarrow I / I^{2} \longrightarrow \circ I^{2} / I^{3} \longrightarrow \circ \rightarrow \cdots
$$

derived from the algebraic $I$-adic tower (0.2). It follows from Theorem 4.3 and arguments analogous to the ones in the preceding proof that it provides a relative injective resolution of $T_{*}$ with respect to the Adams injective class associated to $F_{*}$ (see Remark [5.3). Furthermore, (0.2) is characterized (up to isomorphism) as the Adams resolution associated to it.

Remark 6.9 Having constructed an $I$-adic tower over $T$, we can easily construct one under $T$, of the form:

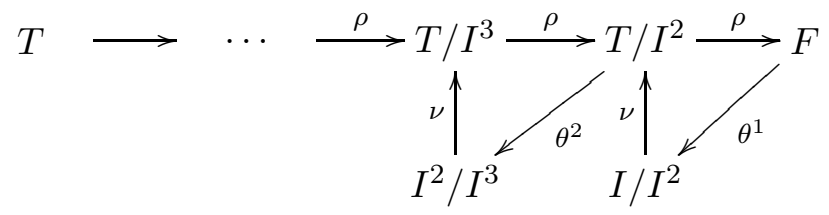

Namely, define spectra $T / I^{s}$ in $\mathscr{D}_{R}$ as cofibres of the canonical maps $I^{s} \rightarrow T$ and construct maps $T / I^{s+1} \stackrel{\rho}{\rightarrow} T / I^{s}$ by making use of the octahedral axiom. It is possible to choose these and compatible maps

$$
\operatorname{holim}_{s} I^{s} \stackrel{\iota}{\longrightarrow} T \stackrel{\rho}{\longrightarrow} \operatorname{holim}_{s} T / I^{s} \longrightarrow \operatorname{holim}_{s} I^{s}
$$

between the homotopy limits so that (6.26) is a cofibre sequence, see [15, Rem. after Prop. 2.2.12]. The diagram of homotopy groups of (6.25) is isomorphic to:

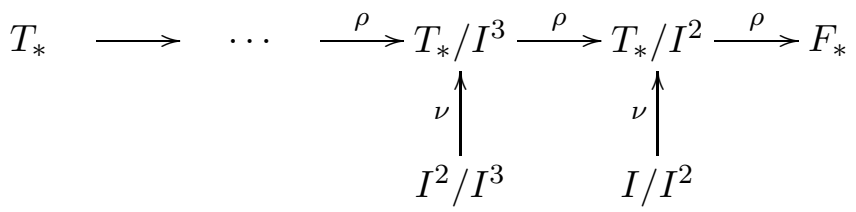


Also, the diagram of $F$-homology groups of 6.25 is isomorphic to $F_{*}^{R}(T) \otimes-$ of the one in Corollary 4.4, with the total gradation.

Notation 6.10 We write $I^{\infty}=\operatorname{holim}_{s} I^{s}$ and $\widehat{T}=\operatorname{holim}_{s} T / I^{s}$ for the homotopy limits of the $I$-adic towers. From Theorem [6.6] we obtain $\widehat{T}_{*} \cong$ $\lim _{s} T_{*} / I^{s}=\left(T_{*}\right)_{I}^{\wedge}$.

We now aim to identify $\widehat{T}$ under certain conditions as a Bousfield localization with respect to $F$ in $\mathscr{D}_{R}$ and in $\mathscr{D}_{\mathbb{S}}$. First, we need to recall some terminology and basic facts about Bousfield localizations.

Let $F$ be a module over a commutative $\mathbb{S}$-algebra $R$. An $R$-module $Z$ is called $F$-acyclic if $F \wedge Z \cong 0$. An $R$-module $X$ is called $F$-local if $[Z, X]_{R}^{*}=0$ for all $F$-acyclic spectra $Z$. A map $f: X \rightarrow Y$ of $R$-modules is called an $F$ equivalence if $F \wedge f$ is an isomorphism. By [15, Thm. 3.2.2], we can associate to $F$ the Bousfield localization and colocalization functors $L_{F}^{R}: \mathscr{D}_{R} \rightarrow \mathscr{D}_{R}$ and $C_{F}^{R}: \mathscr{D}_{R} \rightarrow \mathscr{D}_{R}$ respectively. We write $L_{F}$ and $C_{F}$ if $R=S$. The functors come with natural transformations $i^{R}: 1 \rightarrow L_{F}^{R}$ and $q^{R}: C_{F}^{R} \rightarrow 1$, where 1 is the identity functor. The pair $\left(L_{F}^{R}, i^{R}\right)$ is characterized by the fact that $L_{F}^{R} X$ is $F$-local and that $i_{X}^{R}: X \rightarrow L_{F}^{R} X$ is initial among $F$-local objects under $X$. Also, $i_{X}^{R}$ is an $F$-equivalence for each $X$. Similarly, $\left(C_{F}^{R}, q^{R}\right)$ is characterized by $C_{F}^{R} X$ being $F$-acyclic and $q_{X}^{R}: C_{F}^{R} X \rightarrow X$ being terminal among $F$-acyclic objects over $X$. For each $X$, there are natural cofibrations

$$
C_{F}^{R} X \stackrel{q_{X}^{R}}{\longrightarrow} X \stackrel{i_{X}^{R}}{\longrightarrow} L_{F}^{R} X \rightarrow \Sigma C_{F}^{R} X .
$$

The pair $\left(L_{F}^{R}, i^{R}\right)$ determines $\left(C_{F}^{R}, q^{R}\right)$ by means of these, see [15. Lemma 3.1.6].

The following fact is well-known. We include the proof, as it is quite short.

Lemma 6.11 Let $X$ be an $F$-local in $\mathscr{D}_{R}$. Then $X$ is $F$-local in $\mathscr{D}_{\mathbb{S}}$.

Proof Let $Z$ be an $F$-acyclic spectrum. Then $F \wedge_{R}\left(R \wedge_{\mathbb{S}} Z\right) \cong F \wedge_{\mathbb{S}} Z \cong 0$, so $R \wedge_{\mathbb{S}} Z$ is $F$-acyclic in $\mathscr{D}_{R}$. Therefore, we have

$$
[Z, X]^{*} \cong\left[R \wedge_{\mathbb{S}} Z, X\right]_{R}^{*}=0
$$

which proves that $X$ is $F$-local in $\mathscr{D}_{\mathbb{S}}$.

Recall that the ideal $I \triangleleft T_{*}$ is called invariant in $T_{*}^{R}(T)$ iff $I \cdot T_{*}^{R}(T)=T_{*}^{R}(T) \cdot I$. Here we use the left and the right actions of $T_{*}$ on $T_{*}^{R}(T)$ induced by including $T$ via the unit $\eta_{T}: R \rightarrow T$ as the right or the left factor of $T \wedge_{R} T$ respectively. Similarly, $I$ is defined to be invariant in $T_{*}(T)$ iff $I \cdot T_{*}(T)=T_{*}(T) \cdot I$. 
Proposition 6.12 Assume that the sequence $S$ generating the ideal $I$ is finite. Then the cofibre sequence (6.26)

$$
I^{\infty} \stackrel{\iota}{\longrightarrow} T \stackrel{p}{\longrightarrow} \widehat{T} \longrightarrow \Sigma I^{\infty}
$$

can be identified with the triangle

$$
C_{F}^{R} T \stackrel{q_{F}^{R}}{\longrightarrow} T \stackrel{i_{F}^{R}}{\longrightarrow} L_{F}^{R} T \longrightarrow \Sigma C_{F}^{R} T
$$

arising from localizing $T$ with respect to $F$ in $\mathscr{D}_{R}$. If moreover $I$ is invariant in $T_{*}(T)$, we can view (6.26) in $\mathscr{D}_{\mathbb{S}}$ as

$$
C_{F} T \stackrel{q_{F}}{\longrightarrow} T \stackrel{i_{F}}{\longrightarrow} L_{F} T \longrightarrow \Sigma C_{F} T .
$$

Proof By what was said above, it suffices to exhibit $p: T \rightarrow \widehat{T}$ as the Bousfield localization of $T$ in $\mathscr{D}_{R}$ with respect to $F$ to prove the first statement. By construction, all the objects $T / I^{s}$ lie in the thick subcategory of $\mathscr{D}_{R}$ generated by $F$ and are therefore $F$-local. The class of $F$-local objects is closed under homotopy limits, hence $\widehat{T}$ is $F$-local. To prove that $p$ is an $F$-equivalence, we first show that the composition

$$
F \wedge T \longrightarrow \operatorname{holim}_{s}\left(F \wedge T / I^{s}\right)
$$

of $F \wedge p: F \wedge T \rightarrow F \wedge \widehat{T}$ with the natural map

$$
F \wedge \widehat{T}=F \wedge \operatorname{holim}_{s}\left(T / I^{s}\right) \longrightarrow \operatorname{holim}_{s}\left(F \wedge T / I^{s}\right)
$$

is an equivalence. Consider the Milnor type short exact sequence

$$
0 \longrightarrow \lim _{s}^{1} F_{*+1}^{R}\left(T / I^{s}\right) \longrightarrow\left(\operatorname{holim}_{s} F \wedge T / I^{s}\right)_{*} \longrightarrow \lim _{s} F_{*}^{R}\left(T / I^{s}\right) \longrightarrow 0
$$

of $F_{*}$-modules, see [15, Prop. 2.2.11]. The identification of the $F$-homology of the tower under $T$ in Remark [6.9] together with Corollary 4.4 imply that

$$
\lim _{s} F_{*}^{R}\left(T / I^{s}\right) \cong F_{*}^{R}(T)
$$

and $\lim _{s}^{1} F_{*}^{R}\left(T / I^{s}\right)=0$. Therefore (6.28) is an equivalence. It remains to prove that (6.29) is one as well. Choose a cell approximation $\Gamma F$ of $F$ and a filtration $(\Gamma F)_{k}$ by finite subcomplexes [11, Lemma III.2.3]. Fix a $K$ such that

$$
x_{i}:\left((\Gamma F)_{k}\right)_{*} \longrightarrow\left((\Gamma F)_{k}\right)_{*}
$$

is trivial for $0 \leqslant i \leqslant n$ for all $k>K$. Let $k>K$. It follows by induction over the elements $x_{i}$ of the sequence $S=\left(x_{0}, \ldots, x_{n}\right)$ that

$$
\left((\Gamma F)_{k}\right)_{*}^{R}(F)_{*} \cong\left((\Gamma F)_{k}\right)_{*}^{R}(T) \otimes_{L_{*}} \Lambda_{L_{*}}\left(a_{0}^{\prime}, \ldots, a_{n}^{\prime}\right)
$$


with $\left|a_{i}^{\prime}\right|=\left|x_{i}\right|+1$. Consider the map

$$
\left((\Gamma F)_{k}\right)_{*}^{R}\left(T \wedge Q_{j}\right):\left((\Gamma F)_{k}\right)_{*}^{R}(F) \longrightarrow\left((\Gamma F)_{k}\right)_{*-\left|x_{j}\right|-1}^{R}(F)
$$

induced by the Bockstein $Q_{j}: L \rightarrow \Sigma^{\left|x_{j}\right|+1} L$. Under (6.30), it corresponds to

$$
M_{k *} \otimes \frac{\partial}{\partial a_{j}^{\prime}}: M_{k *} \otimes_{L_{*}} \Lambda_{L_{*}}\left(a_{0}^{\prime}, \ldots, a_{n}^{\prime}\right) \longrightarrow M_{k *} \otimes_{L_{*}} \Lambda_{L_{*}}\left(a_{0}^{\prime}, \ldots, a_{n}^{\prime}\right)
$$

where $M_{k *}=\left((\Gamma F)_{k}\right)_{*}^{R}(T)$. It follows that we can identify the images of the sequence (6.1) under the two functors $\left((\Gamma F)_{k}\right)_{*}(T \wedge-)$ and $M_{k *} \otimes_{L_{*}} L_{*}^{R}(-)$. This implies that $\left((\Gamma F)_{k}\right)_{*}(-)$ of the $I$-adic tower over $T$ is isomorphic to $M_{k *} \otimes_{L_{*}} L_{*}^{R}(-)$ of the Adams resolution associated to (6.1). In particular, we find that

$$
\lim _{s}\left((\Gamma F)_{k}\right)_{*}^{R}\left(T / I^{s}\right) \cong M_{k *}, \lim _{s}^{1}\left((\Gamma F)_{k}\right)_{*}^{R}\left(T / I^{s}\right)=0 .
$$

Thus, a Milnor type short exact sequence implies that

$$
\left(\operatorname{holim}_{s}(\Gamma F)_{k} \wedge T / I^{s}\right)_{*} \cong M_{k *} .
$$

As $(\Gamma F)_{k}$ is finite, it follows that $\left((\Gamma F)_{k} \wedge \widehat{T}\right)_{*} \cong M_{k *}$. Therefore, we have

$$
F_{*}^{R}(\widehat{T}) \cong \operatorname{colim}_{k}\left((\Gamma F)_{k} \wedge \widehat{T}\right)_{*} \cong \operatorname{colim}_{k} M_{k *} \cong F_{*}^{R}(T) .
$$

Now assume that $I$ is invariant in $T_{*}(T)$. By Lemma 6.11 $\widehat{T}$ is $F$-local in $\mathscr{D}_{\mathbb{S}}$. We need to show that $F_{*}(T) \rightarrow F_{*}(\widehat{T})$ is an isomorphism. Similar to before, we first prove that

$$
\left(\operatorname{holim}_{s} F \wedge T / I^{s}\right)_{*} \cong F_{*}(T)
$$

as follows. As $I$ is invariant, the right $T_{*}$-action on $F_{*}(T)$ factors through $F_{*}$. By induction over the elements $x_{i}$ we obtain

$$
F_{*}(F) \cong F_{*}(T) \otimes \Lambda_{F_{*}}\left(a_{0}^{\prime \prime}, \ldots, a_{n}^{\prime \prime}\right)
$$

with $\left|a_{i}^{\prime \prime}\right|=\left|x_{i}\right|+1$. Analogous to the above, we find that

$$
F_{*}\left(T \wedge Q_{j}\right): F_{*}(F) \longrightarrow F_{*-\left|x_{j}\right|-1}(F)
$$

corresponds under (6.32) to:

$$
F_{*}(T) \otimes \frac{\partial}{\partial a_{j}^{\prime \prime}}: F_{*}(T) \otimes \Lambda_{F_{*}}\left(a_{0}^{\prime \prime}, \ldots, a_{n}^{\prime \prime}\right) \longrightarrow F_{*}(T) \otimes \Lambda_{F_{*}}\left(a_{0}^{\prime \prime}, \ldots, a_{n}^{\prime \prime}\right)
$$

Arguing along the same lines as before, this implies (6.31). To prove that

$$
F_{*}(\widehat{T})=F_{*}\left(\operatorname{holim}_{s} T / I^{s}\right) \longrightarrow\left(\operatorname{holim}_{s} F \wedge_{\mathbb{S}} T / I^{s}\right)_{*}
$$

is an isomorphism, we take the sequence $(\Gamma F)_{k}$ from above, pick a $K^{\prime}$ such that

$$
\left((\Gamma F)_{k} \wedge x_{i}\right):\left((\Gamma F)_{k} \wedge_{\mathbb{S}} T\right)_{*} \longrightarrow\left((\Gamma F)_{k} \wedge_{\mathbb{S}} T\right)_{*}
$$

is trivial for $0 \leqslant i \leqslant n$ for all $k>K^{\prime}$ and argue similarly as above. 
The ideal $I_{n}$ is invariant in $B P_{*}(B P)$ 28]. By Landweber exactness of $E(n)_{*}$, we have

$$
E(n)_{*}(E(n)) \cong E(n)_{*} \otimes_{B P_{*}} B P_{*}(B P) \otimes_{B P_{*}} E(n)_{*}
$$

and hence $I_{n}$ is also invariant in $E(n)_{*}(E(n))$. Thus we obtain:

Corollary 6.13 The natural maps $E(n) \rightarrow \widehat{E(n)}$ and $B P \rightarrow \widehat{B P}$, obtained by considering the regular triples $(M U, E(n), K(n))$ and $(M U, B P, P(n))$, are Bousfield localizations with respect to $K(n)$ and $P(n)$ respectively.

Remark 6.14 As $P(n)$ is connective, the second localization can also be interpreted as the $p$-completion of $B P[8$, Thm. 3.1].

We record (6.30) from the proof of Proposition 6.12 and a corresponding statement for cohomology:

Lemma 6.15 If $I$ is invariant in $T_{*}(T)$ and $S=\left(x_{0}, \ldots, x_{n}\right)$ is finite, we have an isomorphism

$$
F_{*}(F) \cong F_{*}(T) \otimes \Lambda_{F_{*}}\left(a_{0}^{\prime}, \ldots, a_{n}^{\prime}\right)
$$

of $F_{*}$-modules, with $\left|a_{i}^{\prime}\right|=\left|x_{i}\right|+1$. If furthermore $F_{*}(T)$ is $F_{*}$-free, there is an isomorphism of $F^{*}$-modules

$$
F^{*}(F) \cong F^{*}(T) \otimes \Lambda_{F^{*}}\left(Q_{0}, \ldots, Q_{n}\right) .
$$

In this case, the exterior algebra $F^{*} \otimes L_{R}^{*}(L)$ maps isomorphically onto the subalgebra $\Lambda_{F^{*}}\left(Q_{0}, \ldots, Q_{n}\right)$ under the natural map of $F^{*}$-algebras

$$
F^{*} \otimes L_{R}^{*}(L) \rightarrow F_{R}^{*}(F) \rightarrow F^{*}(F) .
$$

Remark 6.16 Because of $\widehat{T}_{*} \cong\left(T_{*}\right)_{I}^{\wedge}$, the map $T \rightarrow \widehat{T}$ is an equivalence in case that $T_{*}$ is $I$-adically complete. We have seen in the proof of Proposition 6.12 that $\widehat{T}$ is $F$-local in $\mathscr{D}_{R}$ and hence in $\mathscr{D}_{\mathbb{S}}$ by Lemma 6.11 So we obtain:

Proposition 6.17 Assume that $(R, T, F)$ is a regular triple, with $F_{*} \cong T_{*} / I$. If $T_{*}$ is $I$-adically complete, it follows that $T$ is local with respect to $F$, in $\mathscr{D}_{R}$ as well as in $\mathscr{D}_{\mathbb{S}}$.

In [4, the notation $\widehat{E(n)}$ denotes the spectrum representing completed Johnson-Wilson theory. Recall that the latter is defined on finite spectra $X$ by:

$$
\widehat{E(n)}^{*}(X)=\lim _{s} E(n)^{*}(X) /\left(I_{n}^{s} \cdot E(n)^{*}(X)\right) \cong \lim _{s}\left(E(n)^{*} / I_{n}^{s}\right) \otimes_{E(n)^{*}} E(n)^{*}(X)
$$

This determines the theory uniquely, because its coefficients are linearly compact with respect to the $I_{n}$-adic topology. 
Proposition 6.18 The homotopy limit $\widehat{E(n)}$ of the $I_{n}$-adic tower under $E(n)$ is isomorphic in $\mathscr{D}_{\mathbb{S}}$ to the spectrum representing completed Johnson-Wilson theory.

Proof We claim that it suffices to construct natural morphisms

$$
E(n)^{*} / I_{n}^{s} \otimes_{E(n)^{*}} E(n)^{*}(X) \longrightarrow\left(E(n) / I_{n}^{s}\right)^{*}(X)
$$

for finite complexes $X$ and verify that they are isomorphisms on suspensions of spheres. Namely, we may then take inverse limits over $s$ on both sides. On the left hand side, we obtain completed Johnson-Wilson theory. For the right hand side, we have a Milnor type short exact sequence [15, Prop. 2.2.11]:

$$
0 \longrightarrow \lim _{s}^{1}\left(E(n) / I_{n}^{s}\right)^{*-1}(X) \longrightarrow \widehat{E(n)}^{*}(X) \longrightarrow \lim _{s}\left(E(n) / I_{n}^{s}\right)^{*}(X) \longrightarrow 0
$$

We claim that the $\lim ^{1}$-term vanishes. By [19, Thm. 7.1], it suffices to show that the $E(n)^{*}$-modules

$$
M_{s}^{*}=\left(E(n) / I_{n}^{s}\right)^{*}(X)
$$

are linearly compact with respect to the $I_{n}$-adic topology. By Lemma 6.19 below, the $E(n)^{*}$-action on $M_{s}^{*}$ factors through $E(n)^{*} / I_{n}^{s}$. So $M_{s}^{*}$ is discrete and therefore Hausdorff, as $M_{s}^{*}$ is finitely generated. With [19, §7, Prop. B], it follows that $M_{s}^{*}$ is linearly compact, because $E(n)^{*} / I_{n}^{s}$ is so. Thus, completed

Johnson-Wilson theory and $\widehat{E(n)}^{*}(-)$ coincide on finite complexes. As the coefficients are linearly compact, this implies the claim.

The existence of morphisms as in (6.33) is guaranteed by Lemma 6.19]below.

Lemma 6.19 For any $R$-module $M$, the ideal $I^{s} \triangleleft T_{*}$ lies in the annihilator of $\left(T / I^{s}\right)_{R}^{*}(M)$.

The proof will be given in the next section.

\section{$7 \quad$ Higher Bockstein spectral sequences}

\subsection{Setting up the spectral sequence; convergence}

Let $(R, T, F)$ be a regular triple, with $F=T \wedge L$. In the last section, we have constructed the $I$-adic tower as an Adams resolution of $T$ with respect to $F$ 
in $\mathscr{D}_{R}$. Let $M$ be an $R$-module. If we apply $-\wedge M$ to the tower, we obtain an Adams resolution of $T \wedge M$. It is of the form:

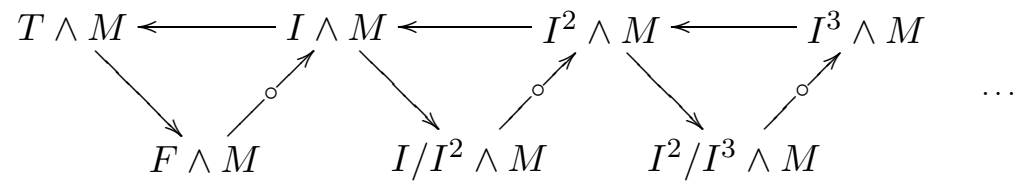

Applying $[Y,-]_{R}^{*}$, where $Y$ is an $R$-module, yields an exact couple, hence a half plane spectral sequence with entering differentials [7, $\S 7$ ].

Let us first recall what we can say about convergence. In general, the homotopy limit holim $_{s} I^{s} \wedge M$ need not be trivial, so we do not have a good convergence behaviour with respect to the target $[Y, T \wedge M]_{R}^{*}$. Boardman shows in [7, $\S 15]$ how this can be remedied. Namely, define $R$-modules $Z^{s}(M)$ by forming cofibre sequences

$$
\operatorname{holim}_{t}\left(I^{t} \wedge M\right) \longrightarrow I^{s} \wedge M \longrightarrow Z^{s}(M)
$$

where the first map is the canonical one. With [15, Rem. after Prop. 2.2.12], we find that

$$
Z^{0}(M) \cong \operatorname{holim}_{s}\left(T / I^{s} \wedge M\right) .
$$

Now construct compatible maps between the $Z^{s}(M)$ via the octahedral axiom. The maps in (7.2) then induce a morphism of (7.1) into the tower

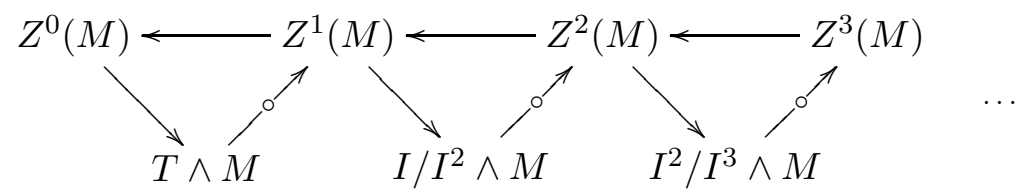

It induces an isomorphism of the respective spectral sequences obtained after applying $[Y,-]_{R}^{*}$, because the $E_{1}$-terms coincide. In the second tower, we have $\operatorname{holim}_{s} Z^{s}(M)=0$. Hence the spectral sequence converges conditionally to

$$
\left[Y, Z^{0}(M)\right]_{R}^{*} \cong\left[Y, \operatorname{holim}_{s}\left(T / I^{s} \wedge M\right)\right]_{R}^{*}
$$

The homotopy $\operatorname{limit}_{\text {holim }}\left(T / I^{s} \wedge M\right)$ can be abstractly characterized as the $F$-nilpotent completion $F_{R}^{\wedge}(T \wedge M)$ of $T \wedge M$ in $\mathscr{D}_{R}$. By this, we mean the obvious generalization to $\mathscr{D}_{R}$ of nilpotent completion with respect to a ring spectrum in $\mathscr{D}_{\mathbb{S}}$, as defined in [8]. In particular, $F_{R}^{\wedge}(T \wedge M)$ is $F$-local for each $M$. This can be seen directly as follows. By induction $T / I^{s}$ and hence $T / I^{s} \wedge M$ are $F$-nilpotent, i.e. lie in the ideal of $\mathscr{D}_{R}$ generated by $F$, in the terminology of [15]. In particular, they are $F$-local. Therefore $F_{R}^{\wedge}(T \wedge M)$ 
is $F$-local, as a homotopy limit of $F$-local $R$-modules. It follows that the canonical map

$$
T \wedge M \rightarrow F_{R}^{\wedge}(T \wedge M)
$$

factors uniquely into the $F$-localization map $i_{F}^{R}: T \wedge M \rightarrow L_{F}^{R}(T \wedge M)$ composed with a map

$$
\varphi_{T \wedge M}: L_{F}^{R}(T \wedge M) \longrightarrow F_{R}^{\wedge}(T \wedge M) .
$$

For each finite $R$-module $M$, the natural map $\widehat{T} \wedge M \rightarrow F_{R}^{\wedge}(T \wedge M)$ is an equivalence, as an induction over the cells of $M$ shows.

If the sequence $S$ generating $I$ is finite, the map $\varphi_{R}$ is an equivalence by Proposition 6.12 If $T=R$, the map

$$
\varphi_{R \wedge M}: L_{F}^{R}(T \wedge M) \cong L_{F}^{R}(M) \longrightarrow F_{R}^{\wedge}(M) \cong F_{R}^{\wedge}(T \wedge M)
$$

is an equivalence for all $M$. Namely, we have Künneth isomorphisms

$$
F_{*}^{R}\left(R / I^{s} \wedge M\right) \cong F_{*}^{R}\left(R / I^{s}\right) \otimes F_{*}^{R}(M)
$$

for all $s$, because $F_{*}^{R}\left(R / I^{s}\right)$ is $F_{*}$-free. Now we have seen in the proof of Proposition 6.12 that $\lim _{s} F_{*}^{R}\left(R / I^{s}\right) \cong F_{*}$ and $\lim _{s}^{1} F_{*}^{R}\left(R / I^{s}\right)=0$. Therefore, we obtain

$$
\lim _{s} F_{*}^{R}\left(R / I^{s} \wedge M\right) \cong F_{*}^{R}(M), \lim _{s}^{1} F_{*}^{R}\left(R / I^{s} \wedge M\right)=0 .
$$

Using a Milnor short exact sequence and the fact that $F$ is a finite $R$-module, the claim follows.

\subsection{The $E_{2}$-term; bicomodules}

If $F_{*}^{R}(Y)$ is $F_{*}$-free, it follows from Proposition 6.2 that the $E_{1}$-term of the spectral sequence is given by:

$$
E_{1}^{s, *}=\left[Y, I^{s} / I^{s+1} \wedge M\right]_{R}^{*} \cong \operatorname{Hom}_{F_{*}}^{*}\left(F_{*}^{R}(Y),\left(I^{s} / I^{s+1} \wedge M\right)_{*}\right)
$$

If $L$ (and hence $F$ ) is commutative as an $R$-ring spectrum and $F_{*}^{R}(T)$ (and hence $\left.F_{*}^{R}(F) \cong F_{*}^{R}(T) \otimes F_{*}^{R}(L)\right)$ is $F_{*}$-flat, the same proposition yields

$$
\left.E_{1}^{s, *} \cong \operatorname{Cohom}_{F_{*}^{R}(F)}^{*}\left(F_{*}^{R}(Y), F_{*}^{R}\left(I^{s} / I^{s+1} \wedge M\right)\right)\right) .
$$

To simplify this expression, we need to gain a better understanding of the $F_{*}^{R}(F)$-comodule structure. We consider the following general setup.

Let $A$ and $B$ be coalgebras over some commutative ring $R$ with coactions $\Delta_{A}$ and $\Delta_{B}$ and counits $\varepsilon_{A}$ and $\varepsilon_{B}$. We write $\otimes$ for $\otimes_{R}$ in the following. Let 
$\tau: A \otimes B \rightarrow B \otimes A$ be a given symmetry isomorphism of $R$-modules with $\tau^{2}=1$. Then we define a left $(A, B)$-bicomodule with respect to $\tau$ to be an $R$-module $M$ which is a left comodule both over $A$ and $B$ in such a way that the diagram

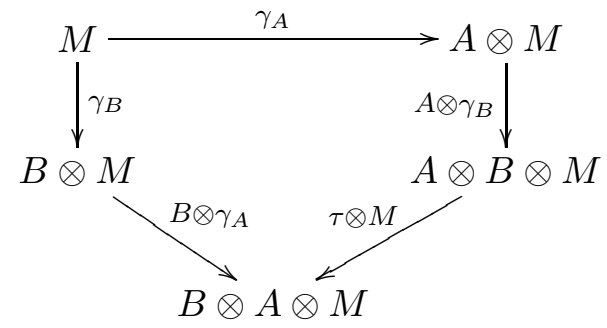

commutes. Here $\gamma_{A}$ and $\gamma_{B}$ denote the given coactions. If we endow $A \otimes B$ with the coproduct

$$
\Delta: A \otimes B \stackrel{\Delta_{A} \otimes \Delta_{B}}{\longrightarrow} A^{\otimes 2} \otimes B^{\otimes 2} \stackrel{A \otimes \tau \otimes B}{\longrightarrow}(A \otimes B)^{\otimes 2},
$$

we find that

$$
M \stackrel{\gamma_{A}}{\longrightarrow} A \otimes M \stackrel{A \otimes \gamma_{B}}{\longrightarrow} A \otimes B \otimes M
$$

defines a coaction of $A \otimes B$ on $M$. To check the coassociativity axiom, we need to verify that the two paths from the top left to the bottom right corner along the outer edges of the following diagram yield the same map:

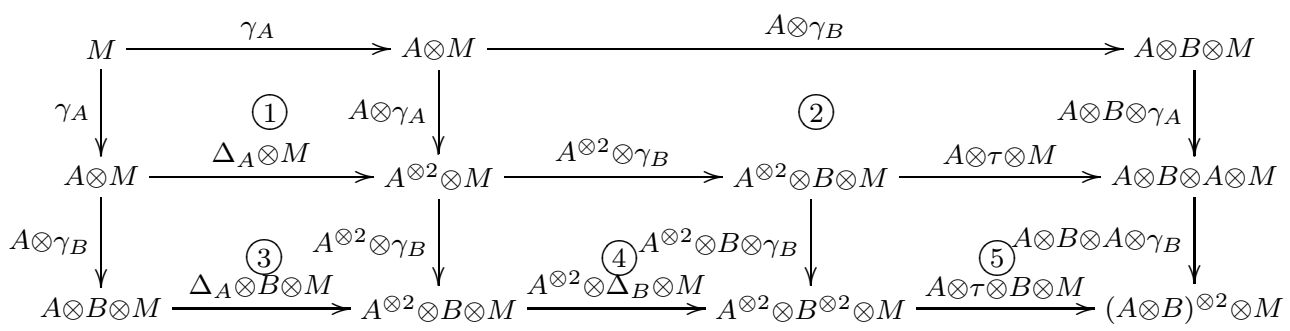

This is the case, as the diagram is built from commuting squares: Squares (1) and (4) commute because $\gamma_{A}$ and $\gamma_{B}$ are coactions; square (2) commutes because $M$ is an $(A, B)$-bicomodule with respect to $\tau$; the remaining two squares commute trivially. The verification of the counit axiom is easy.

Vice versa, an $A \otimes B$-comodule is an $(A, B)$-comodule with respect to $\tau$. Namely, the map

$$
\gamma_{A}: M \stackrel{\gamma}{\rightarrow} A \otimes B \otimes M \stackrel{A \otimes \varepsilon_{B} \otimes M}{\longrightarrow} A \otimes M,
$$

where $\gamma$ denotes the given $A \otimes B$-coaction, defines a coaction of $A$ on $M$. Similarly, we define a coaction $\gamma_{B}$ of $B$. It is easy to check that the coassociativity 
and the counit axioms are satified. To show that $M$ is an $(A, B)$-bicomodule with respect to $\tau$, we check that both

$$
M \stackrel{\gamma_{A}}{\longrightarrow} A \otimes M \stackrel{A \otimes \gamma_{B}}{\longrightarrow} A \otimes B \otimes M
$$

and

$$
M \stackrel{\gamma_{B}}{\longrightarrow} B \otimes M \stackrel{B \otimes \gamma_{A}}{\longrightarrow} B \otimes A \otimes M \stackrel{\tau \otimes M}{\longrightarrow} A \otimes B \otimes M
$$

coincide with the coaction $\gamma$. For (17.5), this can be seen by considering the commutative diagram:

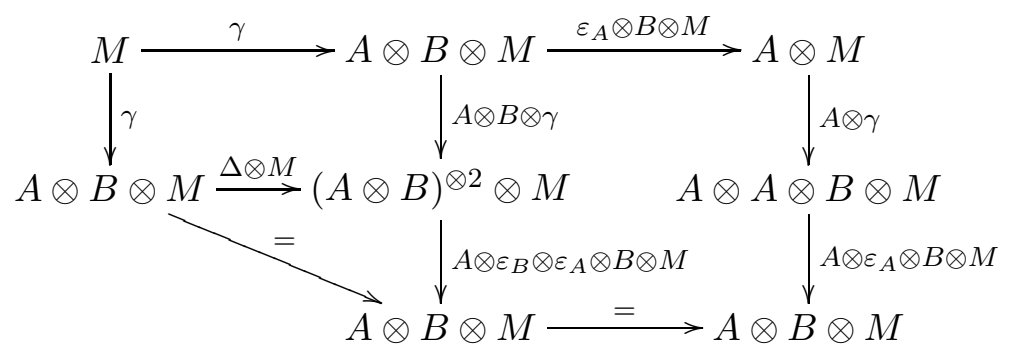

A similar diagram shows that (7.6) is $\gamma$. From now on, we assume that $\tau$ is fixed and don't distinguish between $A \otimes B$-comodules and $(A, B)$-bicomodules with respect to $\tau$.

If $N$ is an $A$-comodule with coaction $\gamma_{A}$, then the composition

$$
B \otimes N \stackrel{B \otimes \gamma_{A}}{\longrightarrow} B \otimes A \otimes N \stackrel{\tau \otimes N}{\longrightarrow} A \otimes B \otimes N
$$

defines an $A$-coaction on $B \otimes N$. Together with the coaction of $B$ given by

$$
B \otimes N \stackrel{\Delta_{B} \otimes N}{\longrightarrow} B \otimes B \otimes N,
$$

$B \otimes N$ can be checked to be an $A \otimes B$-comodule. We say that $B \otimes N$ is obtained by extending the $A$-coaction of $N$ to $A \otimes B$. In a similar way, we can define an extension $A \otimes N^{\prime}$ of a given coaction of $B$ on $N^{\prime}$ to $A \otimes B$. It is then easy to show that we have the following adjunctions for an $A \otimes B$-comodule $M$ :

$$
\begin{aligned}
& \operatorname{Cohom}_{A}(M, N) \cong \operatorname{Cohom}_{A \otimes B}(M, B \otimes N) \\
& \operatorname{Cohom}_{B}\left(M, N^{\prime}\right) \cong \operatorname{Cohom}_{A \otimes B}\left(M, A \otimes N^{\prime}\right)
\end{aligned}
$$

Proposition 7.1 Let $(R, T, F)$ be a regular triple with $F=T \wedge L$. Assume that $L$ (and hence $F$ ) is commutative and that $F_{*}^{R}(T)$ (and hence $F_{*}^{R}(F)$ ) is $F_{*}$-flat. Then there are natural morphisms:

$$
\begin{aligned}
& F_{*}^{R}(M) \longrightarrow F_{*}^{R}(T) \otimes F_{*}^{R}(M) \\
& F_{*}^{R}(M) \longrightarrow F_{*}^{R}(L) \otimes F_{*}^{R}(M)
\end{aligned}
$$


For $M=T$ and $M=L$, they induce coalgebra structures on $F_{*}^{R}(T)$ and $F_{*}^{R}(L)$ respectively. With respect to these, (7.7) and (7.8) define natural coactions on $F_{*}^{R}(M)$. Furthermore, there is a symmetry isomorphism

$$
\tau: F_{*}^{R}(T) \otimes F_{*}^{R}(L) \cong F_{*}^{R}(L) \otimes F_{*}^{R}(T)
$$

with $\tau^{2}=1$ and with respect to which $F_{*}^{R}(M)$ is an $\left(F_{*}^{R}(T), F_{*}^{R}(L)\right)$-bicomodule. The corresponding $F_{*}^{R}(T) \otimes F_{*}^{R}(L)$-coaction coincides under the Künneth isomorphism $F_{*}^{R}(F) \cong F_{*}^{R}(T) \otimes F_{*}^{R}(L)$ with the natural $F_{*}^{R}(F)$-coaction.

We content ourselves with defining the natural maps (7.7), (7.8) and the symmetry $\tau$. The verification of the statements in the proposition is a laborious but straightforward task.

The map (7.7) is defined as the composition

$$
F_{*}^{R}(M) \stackrel{F_{*}^{R}\left(\eta_{T} \wedge M\right)}{\longrightarrow} F_{*}^{R}(T \wedge M) \cong F_{*}^{R}(T) \otimes F_{*}^{R}(M)
$$

where $\eta_{T}: R \rightarrow T$ is the unit of $T$ and the isomorphism is the inverse of:

$$
F_{*}^{R}(T) \otimes F_{*}^{R}(M) \rightarrow\left(T_{0} \wedge L_{0} \wedge T_{1} \wedge T_{2} \wedge L_{1} \wedge M\right)_{*} \rightarrow\left(T_{0} \wedge L_{01} \wedge T_{12} \wedge M\right)_{*}
$$

Here the first map is the canonical one. The purpose of the indices of the various copies of $T$ and $L$ is to indicate in what way these are multiplied under the second map. The right $F_{*}$-action on $F_{*}^{R}(T)$ used to form the tensor product is defined as follows. An element $\gamma_{1} \wedge \gamma_{2} \in(T \wedge L)_{*}=F_{*}$ acts on $x \in F_{*}^{R}(T)$ as

$$
\begin{aligned}
R \stackrel{x}{\rightarrow} F \wedge T \cong(T \wedge L \wedge R) \wedge T \wedge R \stackrel{T \wedge L \wedge \gamma_{2} \wedge T \wedge \gamma_{1}}{\longrightarrow} \\
(T \wedge L \wedge L) \wedge T \wedge T \stackrel{T \wedge \mu_{L} \wedge \mu_{T}}{\longrightarrow}(T \wedge L) \wedge T=F \wedge T
\end{aligned}
$$

where $\mu_{L}$ and $\mu_{T}$ are the products on $L$ and $T$ respectively. Similarly, we define (7.8) as

$$
F_{*}^{R}(M) \stackrel{F_{*}^{R}\left(\eta_{L} \wedge M\right)}{\longrightarrow} F_{*}^{R}(L) \otimes F_{*}^{R}(M) \cong F_{*}^{R}(L \wedge M),
$$

where $\eta_{L}: R \rightarrow L$ is the unit of $L$ and where the isomorphism is the inverse of:

$$
F_{*}^{R}(L) \otimes F_{*}^{R}(M) \rightarrow\left(T_{0} \wedge L_{0} \wedge L_{1} \wedge T_{1} \wedge L_{2} \wedge M\right)_{*} \rightarrow\left(T_{01} \wedge L_{0} \wedge L_{12} \wedge M\right)_{*}
$$

The symmetry $\tau$ is a composition of isomorphisms:

$$
F_{*}^{R}(T) \otimes F_{*}^{R}(L) \cong F_{*}^{R}(T \wedge L) \cong F_{*}^{R}(L \wedge T) \cong F_{*}^{R}(L) \otimes F_{*}^{R}(T)
$$

The first map is (7.9) for $M=L$, the second is induced by the switch $T \wedge L \cong$ $L \wedge T$ and the third is the inverse of (7.10) for $M=T$. 
Remark 7.2 If $T$ is a commutative $\mathbb{S}$-algebra, $F=T \wedge L$ is automatically a $T$-ring spectrum. It is commutative as such if $L$ is commutative as an $R$-ring spectrum. In this case, $F_{*}^{T}(-)$ defines a multiplicative homology theory on $\mathscr{D}_{T}$ taking values in $F_{*}^{T}(F)$-comodules. It can be checked that the coaction of $F_{*}^{T}(F) \cong F_{*}^{R}(L)$ on $F_{*}^{T}(T \wedge M) \cong F_{*}^{R}(M)$ defined in this way is the same as the one considered above.

We leave the verification of the following fact to the reader as well:

Proposition 7.3 For any $R$-module $N$, we have an isomorphism of $F_{*}^{R}(F)$ comodules

$$
F_{*}^{R}(T \wedge N) \cong F_{*}^{R}(T) \otimes F_{*}^{R}(N),
$$

where the coaction on the right hand side is the one obtained by extending the $F_{*}^{R}(L)$-coaction on $F_{*}^{R}(N)$ to $F_{*}^{R}(F)$.

Let us come back to the identification of the $E_{1}$-term of our spectral sequence. It follows from the definition of $I^{s} / I^{s+1}$, the preceding discussion and the fact that $F_{*}^{R}\left(J^{s} / J^{s+1}\right)$ is $F_{*}$-free that we have isomorphisms:

$$
\begin{aligned}
&\left.E_{1}^{s, *} \cong \operatorname{Cohom}_{F_{*}^{R}(F)}^{*}\left(F_{*}^{R}(Y), F_{*}^{R}\left(I^{s} / I^{s+1} \wedge M\right)\right)\right) \\
&\left.=\operatorname{Cohom}_{F_{*}^{R}(F)}^{*}\left(F_{*}^{R}(Y), F_{*}^{R}\left(T \wedge J^{s} / J^{s+1} \wedge M\right)\right)\right) \\
& \cong \operatorname{Cohom}_{F_{*}^{R}(L)}^{*}\left(F_{*}^{R}(Y), F_{*}^{R}\left(J^{s} / J^{s+1} \wedge M\right)\right) \\
& \cong \operatorname{Cohom}_{F_{*}^{R}(L)}^{*}\left(F_{*}^{R}(Y), F_{*}^{R}\left(J^{s} / J^{s+1}\right) \otimes F_{*}^{R}(M)\right)
\end{aligned}
$$

Thus the $E_{2}$-term is the cohomology of the complex obtained by applying the functor $\operatorname{Cohom}_{F_{*}^{R}(L)}^{*}\left(F_{*}^{R}(Y),-\right)$ to the sequence:

$$
0 \rightarrow F_{*}^{R}(M) \rightarrow F_{*}^{R}(L) \otimes F_{*}^{R}(M) \rightarrow F_{*}^{R}\left(\Sigma J / J^{2}\right) \otimes F_{*}^{R}(M) \rightarrow \cdots
$$

We claim that this implies that

$$
E_{2}^{*, *} \cong \operatorname{Coext}_{F_{*}^{R}(L)}^{*, *}\left(F_{*}^{R}(Y), F_{*}^{R}(M)\right) .
$$

To see this, note that (17.11) is the sequence of homotopy groups of

$$
* \longrightarrow F \wedge M \longrightarrow F \wedge L \wedge M \longrightarrow F \wedge \Sigma J / J^{2} \wedge M \longrightarrow \cdots
$$

obtained from (6.1) by applying $F \wedge-\wedge M$. We have noted in the proof of Theorem (6.6) that (6.1) is a relative injective resolution with respect to $L$. Therefore, the sequence (7.12) is split, i.e. exact with respect to the split injective class in $\mathscr{D}_{R}$. It follows that the sequence (7.11) is split over $F_{*}$. This implies that it is a relative injective resolution of $F_{*}^{R}(M)$ over $F_{*}^{R}(L)$ and thus, as $F_{*}^{R}(Y)$ is $F_{*}$-projective, the $E_{2}$-term is as claimed. 


\subsection{The Higher Bockstein spectral sequence}

Let now $X$ be a spectrum, i.e. an object of $\mathscr{D}_{\mathbb{S}}$. By setting

$$
Y=R \wedge_{\mathbb{S}} X, M=R \text { or } Y=R, M=R \wedge_{\mathbb{S}} X
$$

respectively, we obtain the spectral sequences which we call the Higher Bockstein spectral sequences. The following theorem summarizes the above discussion for these cases. Before we state it, we give some comments.

The action of the Bockstein operators $Q_{j} \in L_{R}^{*}(L)$ (Proposition [3.5) on $F_{*}(X)$ and on $F^{*}(X)$ referred to in the statement is obtained from the natural actions of $F^{*}(F)$ via the canonical algebra maps $L_{R}^{*}(L) \rightarrow F_{R}^{*}(F) \rightarrow F^{*}(F)$.

The $F_{*}^{R}(L)$-comodule structure on $F_{*}(X)$ referred to is the one induced by viewing it as $F_{*}(X) \cong F_{*}^{R}\left(R \wedge_{\mathbb{S}} X\right)$. If $F_{*}(F)$ is $F_{*}$-flat, it is given by composing the coaction

$$
F_{*}(X) \longrightarrow F_{*}(F) \otimes F_{*}(X)
$$

with the map $F_{*}(F) \rightarrow F_{*}^{R}(L)$ induced by

$$
\left(T \wedge_{R} L\right) \wedge_{\mathbb{S}}\left(T \wedge_{R} L\right) \rightarrow\left(T_{0} \wedge_{R} L\right) \wedge_{R}\left(T_{1} \wedge_{R} L\right) \rightarrow T_{01} \wedge_{R} L \wedge_{R} L .
$$

The $T$-module $\widehat{T}$ was defined in 6.10 and identified under certain conditions as $L_{F}^{R}(T)$ and as $L_{F}(T)$ in Proposition 6.12. Recall that $F_{*}^{R}(L) \cong \Lambda_{F_{*}}\left(a_{0}, a_{1}, \ldots\right)$ as bialgebras, if $F$ is commutative.

Theorem 7.4 Let $(R, T, F)$ be a regular triple. Then there are conditionally convergent spectral sequences of $T_{*}$-modules, the cohomological and the homological Higher Bockstein spectral sequences, of the form:

$$
\begin{aligned}
& E_{1}^{*, *}=\operatorname{gr}_{I}^{*}\left(T^{*}\right) \otimes F^{*}(X) \Longrightarrow \widehat{T}^{*}(X) \\
& E_{*, *}^{1}=\operatorname{gr}_{I}^{*}\left(T_{*}\right) \otimes F_{*}(X) \Longrightarrow\left(F_{R}^{\wedge}\left(R \wedge_{\mathbb{S}} X\right)\right)_{*}
\end{aligned}
$$

For finite $X$ the target of (17.14) can be identified with $\widehat{T}_{*}(X)$. If $T=R$ and $I$ is finitely generated, then the targets of the spectral sequences are isomorphic to $\left(L_{F}^{R}(R)\right)^{*}(X)$ and $\left(L_{F}^{R}\left(R \wedge_{\mathbb{S}} X\right)\right)_{*}$ respectively. The differential $d_{1}$ of (17.13) is the $\operatorname{gr}_{I}^{*}\left(T^{*}\right)$-linear map determined by

$$
\bigoplus_{j} \bar{v}_{j} \otimes Q_{j}: F^{*}(X) \longrightarrow I / I^{2} \otimes F^{*}(X) .
$$

Explicitly, we have for $\bar{v} \otimes x \in I^{s} / I^{s+1} \otimes F^{*}(X)$

$$
d_{1}(\bar{v} \otimes x)=\sum_{j} \overline{v_{j} v} \otimes Q_{j}(x) .
$$


A similar description holds for the differential $d^{1}$ of (7.14). If $F$ is commutative as an $R$-ring spectrum and $F_{*}^{R}(T)$ is $F_{*}$-flat, the $E_{2}$-term of (7.14) can be expressed as

$$
E_{*, *}^{2} \cong \operatorname{Coext}_{F_{*}^{R}(L)}^{*, *}\left(F_{*}, F_{*}(X)\right) .
$$

If moreover $F_{*}(X)$ is $F_{*}$-free, the $E^{2}$-term of (17.13) is given by

$$
E_{2}^{*, *} \cong \operatorname{Coext}_{F_{*}^{R}(L)}^{*, *}\left(F_{*}(X), F_{*}\right) .
$$

Corollary 7.5 If $F^{*}(X)$ is concentrated in even degrees, the same is true for $\widehat{T}^{*}(X)$. Furthermore, the natural map $\widehat{T}^{*}(X) \rightarrow F^{*}(X)$ is surjective.

We can now give the proof of Lemma 6.19.

Proof of Lemma 6.19 If we cut away in an obvious sense the part of the $I-$ adic tower over $T$ to the left of $T / I^{S}$, we obtain a spectral sequence converging strongly to $\left(T / I^{s}\right)^{*}(X)$. In fact, it collapses at the stage $E_{s}$. Let $\left(F^{l}\right)$ denote the filtration of $\left(T / I^{s}\right)^{*}(X)$ coming from the spectral sequence. We show that $I^{k} F^{l} \subseteq F^{k+l}(*)$. Because $F^{s}$ is trivial, this will prove the lemma. To prove statement $(*)$, note that the $T_{*}$-action on the $E_{1}$-term factors through $T_{*} / I \cong F_{*}$. Hence $I$ acts trivially on the $E_{r}$-term for all $r$. As the spectral sequence collapses, it follows that $I$ acts trivially on $F^{l} / F^{l+1}$. This means that multiplication by an element of $I$ augments the filtration index at least by one. By induction over $k$, this proves $(*)$.

Proposition 7.6 Let $E=\widehat{E(n)}$ and $K=K(n)$ for some prime $p$. The natural map $\left(L_{K}\left(E \wedge_{\mathbb{S}} X\right)\right) \longrightarrow\left(L_{K}^{E}\left(E \wedge_{\mathbb{S}} X\right)\right)$ is an equivalence for any spectrum $X$. Hence the homological Bockstein spectral sequence converges for $R=T=$ $E$ and $F=K$ conditionally to $\left(L_{K}\left(E \wedge_{\mathbb{S}} X\right)\right)_{*}$ for any $X$.

Proof We may identify $K_{E}^{\wedge}\left(E \wedge_{\mathbb{S}} X\right)=\operatorname{holim}_{s}\left(E / I^{s} \wedge_{\mathbb{S}} X\right)$ with the Bousfield localization $L_{K}^{E}\left(E \wedge_{\mathbb{S}} X\right)$ by Theorem 7.4, So the second statement is indeed a consequence of the first. It follows from [13. Thm. 4.2] that the Bousfield localization $L_{K}^{E}(M)$ of an $E$-module $M$ is isomorphic to $M_{I_{n}}^{\wedge}=F_{E}\left(K_{E}\left(I_{n}\right), M\right)$. Here $K_{E}\left(I_{n}\right)$ is the smash product

$$
K_{E}\left(I_{n}\right)=K_{E}\left(v_{0}\right) \wedge_{E} \cdots \wedge_{E} K_{E}\left(v_{n-1}\right),
$$

where the $K_{E}\left(v_{i}\right)$ are defined by the cofibre sequences

$$
K_{E}\left(v_{i}\right) \longrightarrow E \longrightarrow E\left[1 / v_{i}\right]
$$


with $E\left[1 / v_{i}\right] \cong \operatorname{hocolim}\left(E \stackrel{v_{i}}{\longrightarrow} E \stackrel{v_{i}}{\longrightarrow} \cdots\right)$. We may write $F_{E}\left(K_{E}\left(I_{n}\right), M\right) \cong$ $F_{M U}\left(K_{M U}\left(I_{n}\right), M\right)$, where $K_{M U}\left(I_{n}\right)$ is defined as $K_{E}\left(I_{n}\right)$, but $E$ is replaced by $M U$ everywhere. Setting $M=E \wedge_{\mathbb{S}} X$, we find that

$$
L_{K}^{E}\left(E \wedge_{\mathbb{S}} X\right) \cong F_{M U}\left(K_{M U}\left(I_{n}\right), E \wedge_{\mathbb{S}} X\right) .
$$

On the other hand, we have

$$
L_{K}\left(E \wedge_{\mathbb{S}} X\right) \cong F_{\mathbb{S}}\left(\Gamma_{I_{n}}(S) \wedge_{\mathbb{S}} L_{E}(S), L_{E}\left(E \wedge_{\mathbb{S}} X\right)\right)
$$

by [16, Prop. 7.10], where $\Gamma_{I_{n}}(S)$ is the notation from [13] for the homotopy colimit over a certain type of sequence of finite complexes of type $n$. The right hand side is isomorphic to

$$
F_{\mathbb{S}}\left(\Gamma_{I_{n}}(S), E \wedge_{\mathbb{S}} X\right) \cong F_{M U}\left(M U \wedge_{\mathbb{S}} \Gamma_{I_{n}}(S), E \wedge_{\mathbb{S}} X\right) .
$$

Now by [13, Prop. 6.6] the $p$-localizations of $K_{M U}\left(I_{n}\right)$ and $M U \wedge_{\mathbb{S}} \Gamma_{I_{n}}(S)$ are isomorphic. Thus the claim follows.

\subsection{Examples}

We illustrate the Higher Bockstein spectral sequence (HBSS) by considering some examples.

Example 7.7 Classifying spaces of finite groups Let $E=\widehat{E(n)}, I=I_{n}$ and $K=K(n)$ for some $n>0$ and some prime $p$. Assume that $G$ is a finite group and let $B G$ be its classifying space. Ravenel has shown in [27 that $K_{*}(B G)$ is always finitely generated over $K_{*}$. It follows that the HBSS for $(E, E, K)$ converges strongly.

If $G$ is abelian, it follows from the results in [14, Section 5.4] that $E_{*}(B G)$ is finitely generated free over $E_{*}$ and that the natural maps (Lemma 6.19)

$$
E_{*} / I^{S} \otimes_{E_{*}} E_{*}(B G) \rightarrow\left(E / I^{s}\right)_{*}(B G)
$$

are isomorphisms. Therefore the HBSS collapses at $E^{1}$. This follows also from Lemma 7.5] because $K_{*}(B G)$ is concentrated in even degrees [14.

More generally, $K_{*}(B G)$ is trivial in odd degrees for all $G$ in the class of "good groups" as defined in [14]. So $E_{*}(B G)$ is $E_{*}$-free for all these groups. The same is true if $G$ is a symmetric group [17, 31.

However, the classifying space of the $p$-Sylow subgroup of $\mathrm{GL}_{4}\left(\mathbb{F}_{p}\right)$ has nontrivial odd Morava $K(n)$-theory for $p>2$ and $n \geqslant 2$. This was proved by Kriz [20] for $n=2$ and $p=3$ and by Kriz and Lee 21] in the general case. 
Example 7.8 Moore spectra Let $M_{p}$ be the $\bmod p$ Moore spectrum and let us consider the HBSS for the triple $(M U, B P\langle n\rangle, k(n))$ for variety. Write $B=B P\langle n\rangle, k=k(n)$ and $I=I_{n}$. Let $Q_{0}, \ldots, Q_{n-1} \in k_{B}^{*}(k) \subseteq k_{\mathbb{S}}^{*}(k)$ be the Bocksteins associated to the sequence $p, v_{1}, \ldots, v_{n-1}$ and put

$$
L=M U / p \wedge_{M U} \cdots \wedge_{M U} M U / v_{n-1} .
$$

The natural map

$$
k \wedge_{S} M_{p} \simeq k \wedge_{M U} M U / p \longrightarrow k \wedge_{M U} L
$$

induces an injection of homology groups

$$
k_{*}^{S}\left(M_{p}\right) \cong k_{*}^{M U}(M U / p) \cong \Lambda_{k_{*}}\left(a_{0}\right) \longrightarrow \Lambda_{k_{*}}\left(a_{0}, \ldots, a_{n-1}\right) \cong k_{*}^{M U}(L)
$$

where the $a_{j}$ and their formal exterior powers are chosen as in Lemma 3.16, By naturality and the cited lemma, we have $Q_{0}\left(a_{0}\right)=1$. Therefore, the $E^{2}$-term of the spectral sequence is given by $E_{*, *}^{2} \cong \operatorname{gr}_{I}^{*}\left(B_{*}\right) / p$. As it is trivial in odd degrees, it collapses, and we find confirmed that $B_{*}\left(M_{p}\right) \cong B_{*} / p$.

Consider now the mod $p^{2}$ Moore spectrum $M_{p^{2}}$. We have $k_{*}\left(M_{p^{2}}\right) \cong \Lambda_{k_{*}}\left(a_{0}\right)$, but this time $Q_{0}$ is easily seen to act trivially. The fact that for $s>1$

$$
\left(k / I^{S}\right)_{*}\left(M_{p^{2}}\right) \cong k_{*} /\left(I^{s}+\left(p^{2}\right)\right)
$$

implies that $d^{2}\left(\bar{v} \otimes a_{0}\right)=\overline{p^{2} v} \otimes 1$ for any monomial $v$ in $p, v_{1}, \ldots, v_{n-1}$. Hence the spectral sequence collapses at $E_{*, *}^{3} \cong \operatorname{gr}_{I}^{*}\left(B_{*}\right) / p^{2}$, and so $B_{*}\left(M_{p^{2}}\right) \cong B_{*} / p^{2}$.

Example 7.9 Adams-Smith-Toda spectra Let $E=\widehat{E(n)}, K=K(n)$ and $I=I_{n}$ for an odd prime $p$. Then $M_{p}$ admits a self map of degree $2 p-2$, which induces multiplication by $v_{1}$ in $B P$-homology (see e.g. [30, Thm. 1.5]). Define $V$ to be the cofibre. Let $Q_{0}, \ldots, Q_{n-1} \in K_{E}^{*}(K) \subseteq K_{\mathbb{S}}^{*}(K)$ be the Bocksteins corresponding to $p, v_{1}, \ldots, v_{n-1}$. Arguing along similar lines as in the previous example, we find that $K_{*}(V) \cong \Lambda_{K_{*}}\left(a_{0}, a_{1}\right)$ with $Q_{0}\left(a_{0}\right)=1, Q_{1}\left(a_{1}\right)=1$, $Q_{0}\left(a_{0} \wedge a_{1}\right)=a_{1}$ and $Q_{1}\left(a_{0} \wedge a_{1}\right)=-a_{0}$. So we may identify the complex $\left(E_{*, *}^{1}, d^{1}\right)$ with the Koszul complex for the elements $p, v_{1} \in E_{*}$. Hence $E_{*, *}^{2} \cong$

$\operatorname{gr}_{I}^{*}\left(E_{*}\right) /\left(p, v_{1}\right)$, so the spectral sequence collapses and $E_{*}(V) \cong E_{*} /\left(p, v_{1}\right)$, as expected.

\section{References}

[1] A Baker, $A_{\infty}$ structures on some spectra related to Morava $K$-theories, Quart. J. Math. Oxford Ser. (2) 42 (1991) 403-419 MathReview 
[2] A Baker, A Lazarev, On the Adams spectral sequence for $R$-modules, Algebr. Geom. Topol. 1 (2001) 173-199 MathReview

[3] A Baker, B Richter, $\Gamma$-cohomology of rings of numerical polynomials and $E_{\infty}$-structures on $K$-theory, arXiv:math.AT/0304473

[4] A Baker, U Würgler, Liftings of formal groups and the Artinian completion of $v_{n}^{-1} \mathrm{BP}$, Math. Proc. Cambridge Philos. Soc. 106 (1989) 511-530 MathReview

[5] A Baker, U Würgler, Bockstein operations in Morava $K$-theories, Forum Math. 3 (1991) 543-560 MathReview

[6] J M Boardman, Stable operations in generalized cohomology, from: "Handbook of algebraic topology", North-Holland, Amsterdam (1995) 585-686 MathReview

[7] J M Boardman, Conditionally convergent spectral sequences, from: "Homotopy invariant algebraic structures (Baltimore, MD, 1998)", Contemp. Math. 239, Amer. Math. Soc., Providence, RI (1999) 49-84 MathReview

[8] A K Bousfield, The localization of spectra with respect to homology, Topology 18 (1979) 257-281 MathReview

[9] J D Christensen, Ideals in triangulated categories: phantoms, ghosts and skeleta, Adv. Math. 136 (1998) 284-339 MathReview

[10] S Eilenberg, J C Moore, Foundations of relative homological algebra, Mem. Amer. Math. Soc. No. 55 (1965) 39 MathReview

[11] A D Elmendorf, I Kriz, M A Mandell, J P May, Rings, modules, and algebras in stable homotopy theory, Mathematical Surveys and Monographs 47, American Mathematical Society, Providence, RI (1997) MathReview

[12] P G Goerss, M J Hopkins, Moduli spaces of commutative ring spectra, from: "Structured ring spectra", London Math. Soc. Lecture Note Ser. 315, Cambridge Univ. Press, Cambridge (2004) 151-200 MathReview

[13] J P C Greenlees, J P May, Completions in algebra and topology, from: "Handbook of algebraic topology", North-Holland, Amsterdam (1995) 255-276 MathReview

[14] M J Hopkins, N J Kuhn, D C Ravenel, Generalized group characters and complex oriented cohomology theories, J. Amer. Math. Soc. 13 (2000) 553-594 MathReview

[15] M Hovey, J H Palmieri, N P Strickland, Axiomatic stable homotopy theory, Mem. Amer. Math. Soc. 128 (1997) MathReview

[16] M Hovey, N P Strickland, Morava $K$-theories and localisation, Mem. Amer. Math. Soc. 139 (1999) MathReview

[17] J Hunton, The Morava $K$-theories of wreath products, Math. Proc. Cambridge Philos. Soc. 107 (1990) 309-318 MathReview

[18] D Husemoller, J C Moore, Differential graded homological algebra of several variables, from: "Symposia Mathematica, Vol. IV (INDAM, Rome, 1968/69)", Academic Press, London (1970) 397-429 MathReview

[19] C U Jensen, Les foncteurs dérivés de lim et leurs applications en théorie des modules, Springer-Verlag, Berlin (1972) "MathReview 
[20] I Kriz, Morava K-theory of classifying spaces: some calculations, Topology 36 (1997) 1247-1273 MathReview

[21] I Kriz, K P Lee, Odd-degree elements in the Morava $K(n)$ cohomology of finite groups, Topology Appl. 103 (2000) 229-241 MathReview

[22] S Mac Lane, Homology, Classics in Mathematics, Springer-Verlag, Berlin (1995) MathReview

[23] S Mac Lane, Categories for the working mathematician, Graduate Texts in Mathematics 5, Springer-Verlag, New York (1998) MathReview

[24] H Matsumura, Commutative ring theory, Cambridge Studies in Advanced Mathematics 8, Cambridge University Press, Cambridge (1989) MathReview

[25] J P May, $E_{\infty}$ ring spaces and $E_{\infty}$ ring spectra, with contributions by F Quinn, N Ray, and J Tornehave, Lecture Notes in Mathematics 577, Springer-Verlag, Berlin (1977) MathReview

[26] H R Miller, On relations between Adams spectral sequences, with an application to the stable homotopy of a Moore space, J. Pure Appl. Algebra 20 (1981) 287312 MathReview

[27] D C Ravenel, Morava K-theories and finite groups, from: "Symposium on Algebraic Topology in honor of José Adem (Oaxtepec, 1981)", Contemp. Math. 12, Amer. Math. Soc., Providence, R.I. (1982) 289-292 MathReview

[28] D C Ravenel, Complex cobordism and stable homotopy groups of spheres, Pure and Applied Mathematics 121, Academic Press Inc., Orlando, FL (1986) MathReview

[29] D C Ravenel, Nilpotence and periodicity in stable homotopy theory, Annals of Mathematics Studies 128, Princeton University Press, Princeton, NJ (1992) MathReview

[30] L Smith, On realizing complex bordism modules. Applications to the stable homotopy of spheres, Amer. J. Math. 92 (1970) 793-856 MathReview

[31] N P Strickland, Morava E-theory of symmetric groups, Topology 37 (1998) 757-779 MathReview

[32] N P Strickland, Products on MU-modules, Trans. Amer. Math. Soc. 351 (1999) 2569-2606 MathReview

[33] C A Weibel, An introduction to homological algebra, Cambridge Studies in Advanced Mathematics 38, Cambridge University Press, Cambridge (1994) MathReview

[34] S Wüthrich, Multiplicative structures on I-adic towers, in preparation

[35] S Wüthrich, Homology of I-adic towers, arXiv:math.AC/0411187

[36] S Wüthrich, Homology of powers of regular ideals, Glasg. Math. J. 46 (2004) 571-584 MathReview

Department of Pure Mathematics, University of Sheffield

Hicks Building, Hounsfield Road, Sheffield S3 7RH, UK

Email: S.Wuethrich@sheffield.ac.uk

Received: 15 June 2005 Revised: 9 November 2005 